\title{
Mitochondrial Dysregulation in the Pathogenesis of Diabetes: Potential for Mitochondrial Biogenesis-Mediated Interventions
}

\author{
Anna-Maria Joseph, ${ }^{1,2}$ Denis R. Joanisse, ${ }^{3}$ Richard G. Baillot, ${ }^{4}$ and David A. Hood ${ }^{1,2,5}$ \\ ${ }^{1}$ Department of Biology, York University, Toronto, ON, Canada M3J 1 P3 \\ ${ }^{2}$ Muscle Health Research Center (MHRC), York University, Toronto, ON, Canada M3J 1P3 \\ ${ }^{3}$ Division of Kinesiology, Laval University, Québec City, QC, Canada G1K 7P4 \\ ${ }^{4}$ Department of Cardiac Surgery, Laval University, Québec City, QC, Canada G1V 4 G5 \\ ${ }^{5}$ School of Kinesiology and Health Science, York University, Room 302, Farquharson Life Sciences Building, \\ 4700 Keele Street, Toronto, Ontario, Canada M3J 1 P3
}

Correspondence should be addressed to David A. Hood, dhood@yorku.ca

Received 7 June 2011; Accepted 8 September 2011

Academic Editor: Robert A. Harris

Copyright ( 92012 Anna-Maria Joseph et al. This is an open access article distributed under the Creative Commons Attribution License, which permits unrestricted use, distribution, and reproduction in any medium, provided the original work is properly cited.

Muscle mitochondrial metabolism is a tightly controlled process that involves the coordination of signaling pathways and factors from both the nuclear and mitochondrial genomes. Perhaps the most important pathway regulating metabolism in muscle is mitochondrial biogenesis. In response to physiological stimuli such as exercise, retrograde signaling pathways are activated that allow crosstalk between the nucleus and mitochondria, upregulating hundreds of genes and leading to higher mitochondrial content and increased oxidation of substrates. With type 2 diabetes, these processes can become dysregulated and the ability of the cell to respond to nutrient and energy fluctuations is diminished. This, coupled with reduced mitochondrial content and altered mitochondrial morphology, has been directly linked to the pathogenesis of this disease. In this paper, we will discuss our current understanding of mitochondrial dysregulation in skeletal muscle as it relates to type 2 diabetes, placing particular emphasis on the pathways of mitochondrial biogenesis and mitochondrial dynamics, and the therapeutic value of exercise and other interventions.

\section{Introduction}

Type 2 diabetes is the most common form of diabetes accounting for $\sim 90 \%$ of diabetic cases and $\sim 8 \%$ of the total population [1]. Type 2 diabetes is characterized by insulin resistance and is commonly associated with several clinical complications such as hypertension, atherosclerosis, and cardiovascular disease, and these are often collectively referred to as the metabolic syndrome [1]. Although the specific molecular mechanisms underlying type 2 diabetes are not well understood, insulin resistance is believed to result from reductions in glucose transport and phosphorylation and impaired fatty acid metabolism in a number of tissues, notably skeletal muscle $[1,2]$. Specifically, defects in this series of reactions are directly associated with increased levels of plasma and intracellular free fatty acids and alterations in insulin signaling pathways $[3,4]$.
Mitochondria have several functions but are most known for their role as key regulators of metabolic activity within the cell by converting energy from the oxidation of macronutrients to ATP. Mitochondrial activity and function in skeletal muscle is a highly controlled process, under the influence of a variety of nuclear and mitochondrial factors that act as metabolic sensors and can adapt to perturbations in cellular nutrient and energy status. The renewal of mitochondria through the process of biogenesis is vital for maintaining mitochondrial integrity, and a diminished capacity for organelle biogenesis has been implicated in the pathogenesis of several diseases such as aging, neurodegeneration, as well as type 2 diabetes $[1,5]$. Additionally, muscle mitochondrial metabolism is regulated by a group of morphogenesis machinery proteins which are important for mitochondrial fusion and fission events and also for their independent effects on bioenergetics, programmed 
cell death, and autophagy [6]. Defects in mitochondrial biogenesis and morphogenesis factors can impair enzyme activity and reduce the oxidative capacity of the cell leading to insufficient oxidation of lipids and increased intramyocellular lipid (IMCL) levels. The inability of mitochondria to utilize these substrates along with their accumulation within muscle has been associated with impaired insulin signaling pathways and reduced glucose uptake [7]. Elevated IMCLs, in association with the increased production of lipid metabolites such as acyl coenzyme A (CoA), diacylglycerol (DAG), ceramides, and reactive oxygen species (ROS) [2, 8], can affect insulin signaling and contribute to insulin resistance associated with type 2 diabetes. Additionally, skeletal muscle from individuals with type 2 diabetes have a higher percentage of type II fibers and a lower percentage of type I fibers when compared to control individuals $[9,10]$. Type II fibers have a reduced capacity to oxidize fat [11] and possess unique properties that have been shown to potentiate mitochondrial hydrogen peroxide production and oxidative stress [12]. Therefore, there are likely multiple factors that contribute to the stress environment that intensify the mitochondrial dysregulation observed in type 2 diabetes.

The multiplicity of mitochondrial functions has made it a logical target for the study of metabolic diseases, and, given that skeletal muscle represents the major site of insulin-stimulated glucose utilization in the body $[13,14]$, dysregulation of mitochondria is closely associated with insulin resistance and the pathogenesis of type 2 diabetes in muscle. In this paper, we will first discuss key pathways involved in the regulation of mitochondria, with specific attention given to organelle biogenesis, as well as mitochondrial fusion and fission events and their contribution to metabolic perturbations in muscle. In the second part, current therapeutic interventions will be described, with the focus on those related to stimulating mitochondrial biogenesis.

\section{Mitochondrial Biogenesis}

Skeletal muscle is a malleable tissue and can adapt to alterations in energy status and substrate supply in part via its ability to increase the number of mitochondria. Mitochondrial biogenesis is induced by numerous physiological, environmental, and pharmacological stimuli and results from the transcription and translation of genes both in the nuclear and the mitochondrial genomes $[15,16]$. The biogenesis of mitochondria is mediated by changes in many key intracellular events, including transcriptional activation, mRNA stability, posttranslational modification of proteins, and/or alterations in the import and processing of proteins in mitochondria (Figure 1) [17, 18]. These gene products are assembled into functional multisubunit complexes within mitochondria and enhance oxidative capacity and ATP production within the cell. Thus, mitochondria are key regulators of metabolic activity within the cell, and it is these attributes that have made mitochondria a primary focus in the study of metabolic disorders such as type 2 diabetes.

Some of the early studies examining mitochondrial function and insulin resistance reported reduced mitochondrial content and impaired lipid oxidation in skeletal muscle of obese and type 2 diabetic individuals $[19,20]$. The role of mitochondria in obesity and type 2 diabetes has been confirmed by studies examining insulin resistant but otherwise, healthy individuals, with non-insulin-resistant subjects. The insulin-resistant group did not only have higher intramyocellular lipid (IMCL) levels than the control group, but this was associated with a $40 \%$ decrease in both oxidative capacity and ATP levels [21]. Impaired mitochondrial oxidative capacity is also an early feature observed in insulin-resistant offspring of individuals with type 2 diabetes [22]. The altered mitochondrial phenotype observed in human skeletal muscle tissue is retained in myocyte cultures obtained from individuals with type 2 diabetes [23]. Furthermore, an A3243G mutation in the tRNA $^{\text {Leu }}$ gene in mitochondrial DNA (mtDNA) is the cause of maternally inherited diabetes and deafness (MIDD), and individuals with this mutation will likely develop diabetes as they age [24]. These data support the hypothesis that mitochondrial dysfunction may be one of the early events in the pathogenesis of this disease that predisposes an individual to elevated levels of IMCL, lipid metabolites, and insulin resistance.

In order to elucidate the molecular mechanisms that are responsible for the reduction in mitochondrial content and enzyme activity, earlier studies used DNA microarray techniques to examine the gene expression profile of mitochondrial proteins within skeletal muscle of obese and type 2 diabetic patients. These studies found significant alterations in a wide array of genes responsible for glucose uptake, fatty acid oxidation, and oxidative phosphorylation (OXPHOS) $[25,26]$. These include changes in the expression of various nuclear regulatory proteins. Peroxisome proliferatoractivated receptor $\gamma$ (PPAR $\gamma$ ) coactivator-1 (PGC-1) and their related family members (PGC- $1 \alpha$, PGC- $1 \beta$, PRC, and PERC) [27-30] are perhaps among the most well-known regulators of mitochondrial biogenesis. In particular, PGC$1 \alpha$ binds and coactivates transcription factors such as the estrogen-related receptor alpha $(\mathrm{ERR} \alpha)$ and the nuclear respiratory factors 1 and 2 (NRF- 1 and NRF-2) to cause the induction of a broad spectrum of genes involved in substrate metabolism and mitochondrial biogenesis. NRF-1 and NRF2 have been shown to transactivate target genes involved in several mitochondrial processes, including OXPHOS subunits, heme biosynthesis, mitochondrial import machinery, and mtDNA transcription [31-33]. In addition, it has been shown that a dominant negative allele of NRF-1 prevents the ability of PGC- $1 \alpha$ to induce mitochondrial proliferation, confirming the importance of NRF-1 in PGC- $1 \alpha$-driven mitochondrial biogenesis [29].

In skeletal muscle, the importance of PGC- $1 \alpha$ has been reinforced with data from studies in both cell culture, as well as transgenic mouse models, where PGC- $1 \alpha$ levels were experimentally altered. Forced expression of PGC- $1 \alpha$ in cultured muscle cells and cardiac myocytes results in an increase in nuclear and mitochondrial gene expression and mtDNA content $[29,34]$. Animals with increased muscle PGC- $1 \alpha$ have a longer lifespan that is associated with enhanced mitochondrial function, improved insulin sensitivity, and 


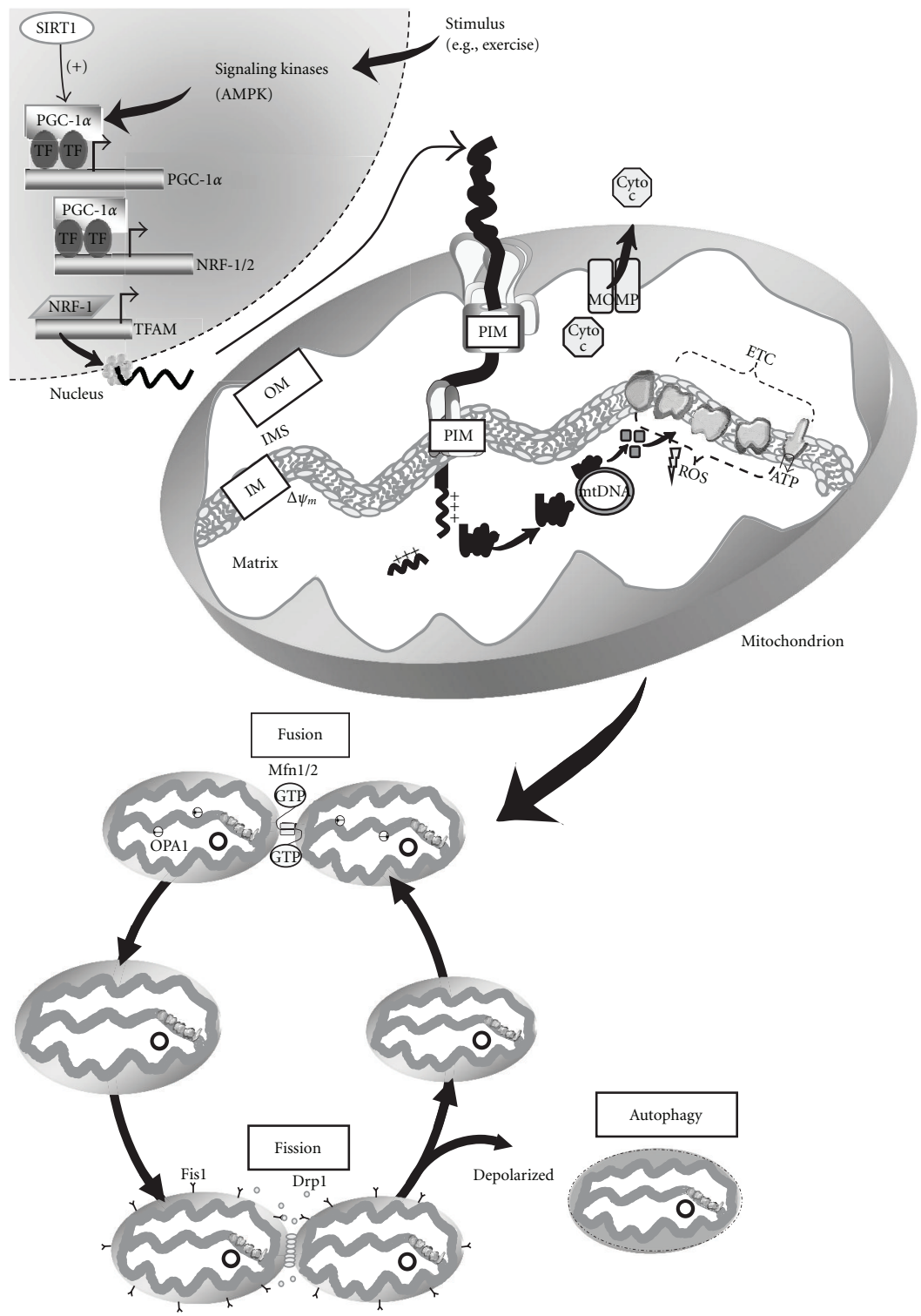

Figure 1: Proposed model of mitochondrial biogenesis. In response to a stimulus such as skeletal muscle contractile activity or exercise, intracellular $\mathrm{Ca}^{2+}$ levels, as well as AMP levels, increase leading to the activation of signaling molecules including AMP-activated protein kinase (AMPK). These signaling pathways converge and interact primarily with the transcriptional coactivator peroxisome proliferatoractivated receptor- $\gamma$ coactivator- $1 \alpha$ (PGC- $1 \alpha$ ) which is a master regulator of mitochondrial biogenesis. PGC- $1 \alpha$ activates its own expression, as well as the expression of the nuclear respiratory factor-1 and 2 (NRF-1/2). Additionally, PGC-1 $\alpha$ has recently been shown to be deacetylated and activated by the longevity protein sirtuin 1 (SIRT1). NRF-1 and NRF-2 bind and upregulate the expression of nuclear genes encoding mitochondrial proteins (NUGEMPs), as well as the expression of mitochondrial transcription factor A (Tfam). Tfam along with other newly transcribed NUGEMPS are targeted and imported into mitochondrial subcompartments via the protein import machinery (PIM). Within the matrix, Tfam binds to mtDNA and regulates the expression of the 13 mitochondrial DNA (mtDNA) gene products. These proteins are assembled into multisubunit enzyme complexes within the electron transport chain (ETC) and mediate oxidative phosphorylation (OXPHOS) and the production of ATP. Thus, coordinated expression regulated by the two genomes allows for the proper assembly and expansion of the mitochondrial reticulum leading to mitochondrial proliferation and increased mitochondrial number/content. Another important product of the ETC is reactive oxygen species (ROS) that are associated with the mitochondrial membrane potential $\left(\Delta \psi_{\mathrm{m}}\right)$. Elevated levels of ROS have been shown to activate mitochondrial outer membrane permeabilization (MOMP) and the release of proapoptotic factors such as cytochrome c (Cyt c) into the cytosol that can subsequently activate caspase-dependent signaling cascades leading to mitochondrially-mediated apoptosis. Furthermore, organelle biogenesis requires a continuous cycle of fusion and fission events. Mitochondrial fusion of the outer and inner mitochondrial membranes is mediated by the GTPase proteins, mitofusin 1 and 2 (Mfn1 and Mfn2) and OPA1, respectively. Conversely, mitochondrial fission requires Drp1 and Fis1 which assemble at fission sites on the mitochondrial membrane and induce membrane division. It has been proposed that fission can lead to mitochondria with different $\Delta \psi_{\mathrm{m}}$ and that damaged or depolarized organelles will exit the fusion and fission cycle and will be removed through autophagy. 
reduced oxidative damage and also show resistance to agerelated weight gain [35]. Furthermore, overexpression of PGC- $1 \alpha$ in mice results in a partial fiber-type transition from white muscle with mostly glycolytic fibers to muscle that appears red and has a high oxidative capacity [36]. This fiber-type conversion coincides with the activation of calcineurin signaling cascades, the coactivation of myocyteenhancer factor 2 (MEF2) by PGC-1 $\alpha$, and the induction of slow gene expression pathways. Calcium- $\left(\mathrm{Ca}^{2+}{ }^{2}\right)$ dependent PGC- $1 \alpha$ activation was further confirmed in skeletal muscle from transgenic mice overexpressing a constitutively active form of the calcium/calmodulin-dependent protein kinase IV (CaMKIV). These mice displayed increased mtDNA copy number and an upregulation of several enzymes that are involved in fatty acid oxidation and OXPHOS [37]. Additionally, upregulation of PGC- $1 \alpha$ mRNA and protein with acute and chronic exercise in both animals and humans leads to an increased mitochondrial content through the induction of NRF proteins and mitochondrial transcription factor A (Tfam) [38-40].

Regarding metabolic disorders, PGC- $1 \alpha$ mRNA levels are reduced in certain cohorts of obese and type 2 diabetic individuals $[25,26]$, and, in some populations, polymorphisms in the PGC- $1 \alpha$ gene have been linked to a predisposition for type 2 diabetes $[41,42]$. PGC- $1 \alpha$ induces the expression of the insulin-sensitive glucose transporter (GLUT4) by interacting and coactivating the MEF2 transcription regulator [43]. Furthermore, the tissue-specific knockout of Tfam in pancreatic $\beta$ cells leads to the development of diabetes that is associated with a loss of mtDNA and impaired oxidative capacity [44]. Despite these findings, the importance of PGC- $1 \alpha$ and other mitochondrial regulators of biogenesis in insulin resistance and type 2 diabetes has remained controversial. This is because several studies have shown elevated IMCL levels and reduced mtDNA content in the absence of changes in PGC- $1 \alpha$ expression (mRNA or protein) or other PGC- $1 \alpha$-related target genes [22, 45, 46]. In addition, several studies of muscle-specific PGC$1 \alpha$ and/or PGC- $1 \beta$ null mice have demonstrated normal glucose tolerance and insulin sensitivity $[47,48]$. These studies suggest that alternate mechanisms may also regulate mitochondrial content in metabolic diseases. Clearly more work is required in this area to obtain a better understanding of the molecular pathways mediating insulin sensitivity in both healthy muscle, as well as muscle with metabolic dysfunction.

Another clue into the molecular function of PGC- $1 \alpha$ comes with the recent finding that PGC- $1 \alpha$ is present within mitochondria and specifically localized in a complex with Tfam in mtDNA nucleoids [49]. This surprising finding is also confirmed in animals where, following an acute bout of exercise, PGC- $1 \alpha$ protein was increased in both the nuclear and mitochondrial subfractions [50]. These preliminary studies suggest that PGC- $1 \alpha$ coactivates mitochondrial transcription in both the nucleus and mitochondria and indicates the potential of PGC- $1 \alpha$ as being a central messenger of nuclear-mitochondrial crosstalk during cellular stress.

Recently, another family of proteins has emerged as crucial regulators of mitochondrial activity and cellular energy metabolism. Sirtuins are a group of class III histone/protein deacetylases that are primarily known for their involvement in promoting lifespan in a number of organisms including yeast, flies, and mice, and they accomplish this by the acetylation and deacetylation of target genes [51]. To date, seven sirtuin mammalian homologs have been identified (SIRT1-7), three of which are mainly localized to mitochondria (SIRT3, SIRT4, SIRT5) [51, 52]. SIRT1 is a NAD-dependent deacetylase that is widely expressed in mammalian cells and activated in response to cellular stress conditions such as with exercise, caloric restriction (CR), and starvation [53-57]. Transgenic mice overexpressing SIRT1 have similar physiological and behavioral phenotypes as calorie restricted mice. These animals have less body fat, are more metabolically active, and display improved insulin sensitivity and glucose tolerance [58]. The CR-mediated phenotype is dependent on SIRT1, since knockout of this gene diminishes these adaptations [59]. Studies investigating the role of SIRT1 in diabetes and skeletal muscle are limited. In cardiac muscle of Otsuka Long-Evans Tokushima fatty (OLETF) rats, SIRT1 levels were lower in these animals than in control rats. Treatment with pioglitazone, a PPAR $y$ agonist, enhanced SIRT1 expression [60]. Similar attenuations in SIRT1 levels were reported in adipocytes from ob/ob mice [61] and adipose tissue from obese women [62]. SIRT1 gainof-function studies in various models of insulin resistance and diabetes have revealed improved glucose tolerance and decreased energy expenditure that is due to lower hepatic glucose production and increased adiponectin levels [63] suggesting that SIRT1-mediated longevity may be related to improvements in insulin sensitivity.

SIRT1 regulates AMP activated-protein kinase (AMPK), a key energy-sensing molecule that is activated during conditions of low ATP and high AMP (increased AMP/ATP ratio) such as with exercise [64] and CR [65]. Interestingly, both SIRT1 and AMPK together or independently activate PGC- $1 \alpha$ to induce the expression of genes involved in glucose and fatty acid metabolism and restore ATP levels [66-69]. AMPK activity levels decline with aging, and this is associated with reduced insulin sensitivity and diminished fatty acid oxidation, suggesting that AMPK is an important regulator of mitochondrial metabolism in muscle [70, 71].

More recently, another histone/protein deacetylase has gained increasing attention as a key regulator of metabolic activity in muscle. SIRT3 contains a cleavable $\mathrm{N}$-terminal mitochondrial targeting signal that permits its import into mitochondrial subcompartments [51]. SIRT3 levels were diminished with aging and a high fat diet $[65,72]$ and increased in response to exercise, CR, and fasting $[65,72$, 73]. It is likely that, similar to SIRT1, SIRT3 is a key regulator of muscle adaptation since it also targets PGC- $1 \alpha$ and influences mitochondrial transcriptional regulation in muscle $[65,74]$.

It is important to remember that skeletal muscle contains two subpopulations of mitochondria, subsarcolemmal (SS) and intermyofibrillar (IMF) mitochondria, that display key differences in biochemical and functional properties [75]. These differences are crucial because it has been proposed 
that, due to their close proximity to myonuclei, SS mitochondria may be important for driving processes at the cell surface, including the propagation of insulin signaling pathways, fatty acid oxidation, and glucose transport [75, 76]. In contrast, IMF mitochondria are thought to provide energy for muscle contractions [75]. In both SS and IMF subfractions, electron transport chain (ETC) activity was reduced in obese and type 2 diabetic subjects when compared to lean subjects [77]. Interestingly, the decrement in enzyme activity was more pronounced in the SS, compared to the IMF subfraction, suggesting that SS mitochondria may be more readily affected in states of altered glucose homeostasis. These data are consistent with previous findings showing that SS mitochondria are more labile in response to metabolic changes $[78,79]$.

\section{Mitochondrial Fusion and Fission}

The adaptability of skeletal muscle is also associated with mitochondrial morphological plasticity. Mitochondria are dynamic and readily adapt to changes in cellular energy demands through network remodeling and continuous fusion and fission $[5,80]$. Under normal conditions, mitochondrial fusion results in the formation of an interconnected mitochondrial network that allows the mixing and redistribution of proteins and mtDNA and which has been hypothesized to prevent the accumulation of mutated or damaged mtDNA in a cell $[5,81,82]$. In contrast, mitochondrial fission leads to mitochondria with a fragmented morphology facilitating the segregation of damaged mitochondria that can then be targeted for degradation via autophagy [5]. Although the molecular mechanisms mediating these morphological changes remain largely unknown, recent studies have identified distinct fusion and fission machinery that appear to regulate these processes $[5,81,82]$.

The fusion of mitochondria in mammalian cells is mediated by several proteins, the most well known being the nuclear-encoded dynamin-related guanosine triphosphatases (GTPase), mitofusin 1 (Mfn1), and mitofusin 2 (Mfn2). Although these mitofusins share greater than $70 \%$ homology, they have different GTPase activity levels and display distinct expression patterns, with Mfn2 present in higher amounts in tissues such as heart and skeletal muscle [83, 84]. Additionally, mutations in Mfn2 cause CharcotMarie-Tooth (CMT) disease-type 2A, the most common form of CMT disease and an inherited neuropathy leading to progressive weakness and sensory loss [85, 86]. While mitofusins are responsible for tethering and fusion of the outer mitochondrial membrane, another dynamin family GTPase, Optic Atrophy 1 (OPA1), is required for inner membrane fusion. OPA1 was first identified through its involvement in the neurodegenerative disease known as autosomal dominant optic atrophy (ADOA) [87]. Alternative splicing of OPA1 produces multiple variants that are distinctly present in different species and tissues [88]. Furthermore, posttranslational modification of OPA1 by mitochondrial processing peptidase (MPP) results in different length isoforms that vary based on their localization and function [89, 90]. Muscle-specific Mfn1 and Mfn2 knockout mice with a diminished capacity for mitochondrial fusion have impaired mitochondrial function and a loss of muscle mass that are associated with increased mtDNA point mutations and deletions and severe mtDNA depletion [91]. Similarly, silencing of OPA1 in mammalian cells blocked mitochondrial fusion and resulted in mitochondrial fragmentation, decreased OXPHOS, poor cell growth, and reduced mitochondrial membrane potential $\left(\Delta \psi_{\mathrm{m}}\right)$ [92]. These data indicate that mitochondrial fusion is important for maintaining the integrity of the organelle by allowing the intramitochondrial exchange of damaged mitochondria, preventing their localization within specific organelles and their accumulation within the cell.

Mitochondrial homeostasis is also regulated by fission machinery such as dynamin-related protein 1 (DLP1/Drp1) and fission protein 1 (Fis1). Drp1 is a dynamin-related GTPase that is found in the cytosol and recruited by Fis1 to scission sites on the mitochondrial outer membrane to induce mitochondrial fission. Downregulation of Drp1 in HeLa cells leads to a loss in mtDNA, reduced mitochondrial respiration, and higher levels of ROS, all of which are associated with mitochondrial dysfunction [93]. Also, blocking fission by reducing the levels of Drp1 and Fis1 genes in a human cell line with a mtDNA mutation exacerbates the abundance of mutant mtDNA compared with wild-type mtDNA [94]. These studies imply that mitochondrial fusion and fission are involved in mtDNA quantity and quality control and are required for the maintenance of healthy organelles.

It is also becoming more and more apparent that mitochondrial morphology is directly linked to mitochondrial function and substrate utilization. Cells with a high mitochondrial fusion capacity display interconnected mitochondria associated with increased OXPHOS, while cells with high mitochondrial fission have fragmented mitochondria and rely more on anaerobic metabolism pathways for energy [81]. A hallmark of mitochondrial biogenesis is an expansion of the mitochondrial reticulum, thereby allowing the propagation of signaling pathways and the mixing of metabolites. A perfect example of this adaptation in muscle occurs with exercise, whereby repeated bouts of an exercise stimulus lead to muscle adaptations. Substantial evidence in the last decade has shown the involvement of mitochondrial network remodeling in these exercise-induced adaptations. The expression levels of Mfn1, Mfn2, and Fis1 have been shown to be increased in skeletal muscle following an acute bout of exercise in both animals and humans $[95,96]$. Additionally, transcript levels of mitochondrial dynamics proteins are upregulated with endurance exercise in healthy subjects, and these are closely correlated with muscle OXPHOS activity and PGC- $1 \alpha$ mRNA content [97]. An important finding of these studies is that fusion and fission machinery respond rapidly to an exercise stimulus and that the levels of these proteins are dependent on the type of exercise $[95,96]$.

Given the involvement of mitochondrial dynamics in muscle metabolism, perturbations in the fusion-fission balance caused by changes in the levels of the molecular machinery have been shown to lead to abnormal mitochondrial morphology and to negatively impact mitochondrial 
function. Mitochondrial gene expression profiles and function are associated with changes in overall mitochondrial morphology in skeletal muscle from diabetic rats [83], as well as in humans with type 2 diabetes $[83,98]$. In particular, mitochondria in skeletal muscle from type 2 diabetics are smaller in size than mitochondria present in lean subjects, and they also contain abnormal cristae structure that would suggest defects in the inner membrane [83, 98, 99]. In our laboratory, we have found reduced levels of fusion proteins Mfn2 and Opa1, but no alterations in fission proteins Drp1 or Fis1 in skeletal muscle from type 2 diabetic individuals (Figure 2(a)). Similar reports of decreased Mfn2 expression and aberrant mitochondrial morphology have been documented by other studies in type 2 diabetics [98, 99].

Loss of function of the fusion protein Mfn2 leads to impaired mitochondrial metabolism and is associated with reduced mitochondrial oxygen consumption, membrane potential, and glucose metabolism in a variety of tissues $[83,92,100]$. In contrast, Mfn2 gain of function increases substrate oxidation and improves mitochondrial metabolism in HeLa cells [100]. Additionally, similar to other key metabolic factors, Mfn1 and Mfn2 are increased in response to weight loss and exercise in healthy, obese, and type 2 diabetic individuals $[95,101]$ and are increased also in mice subject to CR in mice [102]. This is dependent primarily on PGC- $1 \alpha$ and ERR $\alpha$ transactivation of the Mfn2 promoter, suggesting that fusion is an important signaling event for mitochondrial biogenesis and healthy insulin signaling in muscle [95, 103]. Additionally, reduced levels of OPA1 are associated with insulin resistance in several cell types [104], as well as in human fibroblasts from patients with ADOA with impaired OXPHOS and reduced mitochondrial fusion events [105]. These findings are in keeping with the observation that mitochondrial fusion is an important signaling event for mitochondrial biogenesis in muscle. For the most part, the regulatory mechanisms and the functional importance of these protein changes in obesity and type 2 diabetes remain enigmatic, and more work is required to elucidate the regulatory factors of the other fusion and fission machineries.

3.1. Mitochondrial Dynamics and Cell Death. The finding that aberrant mitochondrial morphology is present in cells undergoing apoptosis has led to the examination of the possible link between mitochondrial fusion and fission processes and programmed cell death. A hallmark of apoptosis is mitochondrial outer membrane permeabilization (MOMP) and the release of cytochrome $\mathrm{c}$ that activates proapoptotic signaling cascades. This results in the fragmentation of DNA [106]. Imaging experiments have demonstrated that mitochondrial fragmentation occurs concurrently with MOMP. This is primarily due to fission, since either the inhibition of fission proteins or upregulation of fusion proteins can delay or prevent mitochondrial fragmentation, along with the induction of proapoptotic signaling events including MOMP, cytochrome c release, and cell death $[89,107]$.
Studies conducted to elucidate the molecular mechanisms responsible for the association between mitochondrial morphology and apoptosis revealed a physical interaction between morphogenesis proteins and the apoptosis machinery. In particular, proapoptotic proteins such as Bax and Bak interact with Mfn proteins [108, 109], and similar findings were reported between the fission machinery and the Bcl-2 family of proteins $[110,111]$. It is highly likely that the physical interaction between apoptotic proteins and mitochondrial morphogenesis proteins is another means of regulating cell death. However, not all studies are consistent with this theory, and more work is required to clearly show whether mitochondrial fusion and fission are an important part of the apoptosis signaling cascade. Nonetheless, altering levels of fusion and fission events can confer protection to the cell, and this introduces a potential mechanism of decreasing apoptotic susceptibility through the modulation of the fusion and fission machinery.

Mitochondria represent the major source of ROS production within the cell and increased levels of ROS are a likely culprit in a variety of pathophysiological conditions, including type 2 diabetes. Increased ROS production was associated with altered mitochondrial morphology in myotubes cultured in high glucose conditions, as well as in diet-induced diabetic mice [112]. Furthermore, increased oxidative stress within mitochondria arising from impaired oxidative metabolism may contribute to greater lipid peroxidation and damage to cell membranes and DNA, activating a cascade of signaling events that further exacerbate the severity of the disease [113]. A number of studies have found that altering mitochondrial fusion and fission events can influence ROS production in mitochondria. For instance, increasing mitochondrial fusion or decreasing mitochondrial fission during hyperglycemia can prevent mitochondrial fragmentation and reduce ROS levels in the cell [107]. Treatment of cells with hydrogen peroxide, antimycin A, or rotenone to increase ROS production resulted in fragmented mitochondria [107, 114], while addition of an antioxidant reduced ROS levels and inhibited mitochondrial fragmentation [115]. The effect of mitochondrial fusion and fission machinery on apoptosis is likely associated with its ability to modulate ROS levels. Thus, mitochondrial dynamics is a relatively new field in mitochondrial biology, and despite growing progress, many questions still remain regarding how alterations in mitochondrial shape affect mitochondrial bioenergetics, morphology, and ROS production, as well as the sequential order of these events in skeletal muscle with obesity and type 2 diabetes.

Furthermore, a growing area of interest in the field of metabolic diseases is the study of obesity in the elderly, the prevalence of which has considerably increased in the last few years $[116,117]$. As we age, there is a progressive decline in muscle mass and strength, as well as an increased fat mass. This combination of sarcopenia and obesity has recently been defined as "sarcopenic obesity" $[116,117]$. While the molecular mechanisms mediating a loss of muscle mass in obesity and type 2 diabetes are unknown, several theories, including increased apoptosis, have been brought forward. Recently, 


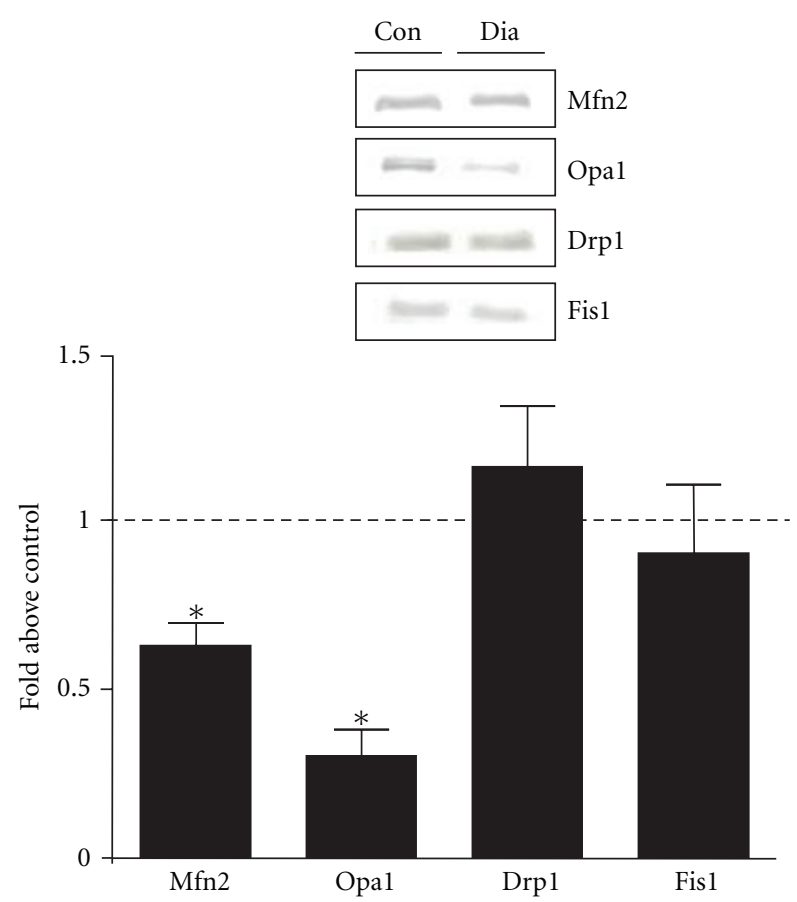

(a)

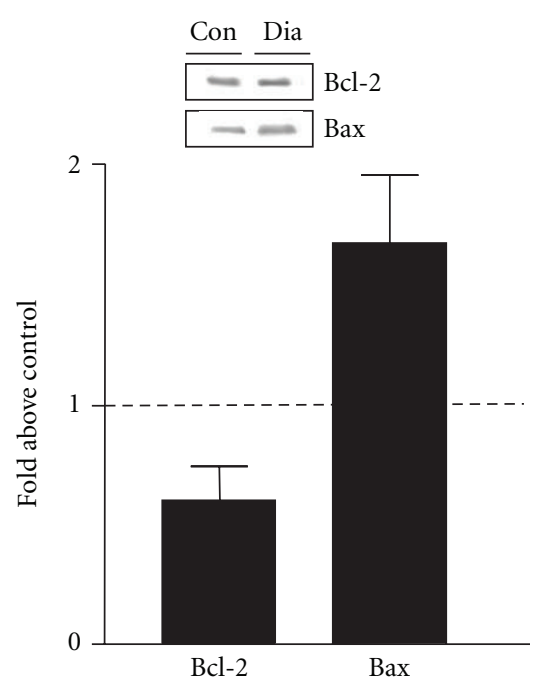

(b)

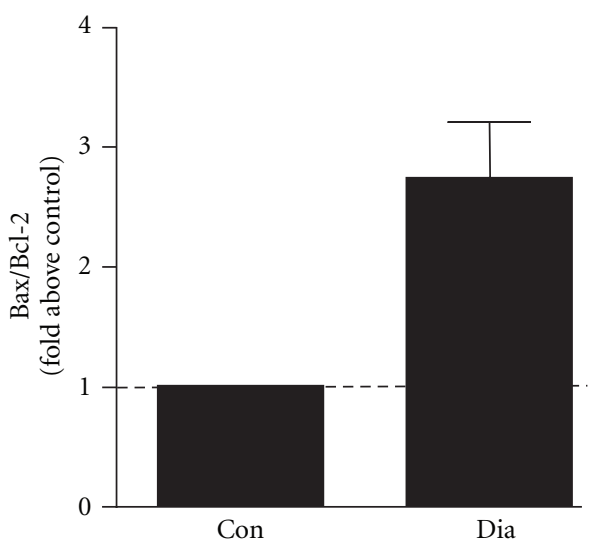

(c)

Figure 2: (a) Mitochondrial morphology proteins in type 2 diabetes. The research volunteers that participated in this study were obese subjects with type 2 diabetes undergoing coronary bypass surgery and were all male between 48 and 75 years of age. Biopsies from the vastus medialis muscle were removed from both control and type 2 diabetic subjects from within incisions of the inner thigh and protein analyses performed. The protocol was approved by the Medical Ethics Committees of Laval University and Laval Hospital, and all subjects provided informed written consent. Representative western blots of fusion proteins Mfn2 and OPA1 and fission proteins Drp1 and Fis1 from the vastus medialis muscle of control (Con) and type 2 diabetic subjects (Dia). A summary of repeated experiments is shown below with values expressed as a fold over control. Values are means \pm SE; $n=4-9 ;{ }^{*} P<0.05$ versus Con. (b) Indicators of apoptotic susceptibility in type 2 diabetes. Western blots of the antiapoptotic protein Bcl-2 and the proapoptotic protein Bax in vastus medialis muscle of Con and Dia individuals and the graphical representation of the data is shown below. Values are means \pm SE; $n=4-9$. (c) The ratio of Bax/Bcl-2 in Dia subjects when compared to Con.

Sishi et al. [118] have demonstrated that, as a result of dietinduced obesity, skeletal muscle of adult rats displayed muscle atrophy along with increased apoptosis. This was evident by increased levels of caspase- 3 and poly(ADP-ribose) polymerase (PARP), two hallmark features of cell death. Additionally, electron micrographs of muscle fibers from obese and type 2 diabetic individuals display an altered mitochondrial structure and fragmented mitochondria, indicating the potential involvement of apoptosis [99]. We have obtained similar results of increased apoptotic signaling, with a trend toward higher levels of the proapoptotic protein Bax and lower levels of the antiapoptotic protein Bcl-2 observed in 
muscle from type 2 diabetics (Figure 2(b)). This indicates a greater Bax/Bcl-2 ratio (Figure 2(c)) and suggests that muscle from type 2 diabetics has a greater susceptibility to apoptosis when compared to healthy individuals. Although these studies point to the involvement of apoptosis in sarcopenic obesity, much more research is required to characterize apoptotic signaling pathways in obesity and type 2 diabetes.

3.2. Mitochondrial Dynamics and Autophagy. Mitochondrial dynamics are also involved in another form of cell death known as autophagy. Autophagy protects the cell through the selective degradation and recycling of organelles such as mitochondria by lysosomal machinery $[119,120]$. Under normal conditions, autophagy prevents the accumulation of damaged mitochondria within the cell that can trigger apoptotic pathways and irreversible cell death. Autophagy is particularly important for postmitotic tissues such as skeletal muscles that are exposed to high levels of oxidative stress and that do not have an inherently high capacity for regeneration $[119,120]$.

Recent studies have shed light on the function and regulation of autophagic pathways in skeletal muscle and have implicated the involvement of mitochondrial fission. The monitoring of individual mitochondria in cultured cells has revealed that daughter mitochondria generated by mitochondrial fission display different mitochondrial membrane potentials $\left(\Delta \psi_{\mathrm{m}}\right)$, with one mitochondrion possessing a high $\Delta \psi_{\mathrm{m}}$ and the other a low $\Delta \psi_{\mathrm{m}}$. Interestingly enough, depolarized mitochondria with a lower $\Delta \psi_{\mathrm{m}}$ were selectively degraded by autophagy and displayed a lower fusion capacity when compared to mitochondria with a high $\Delta \psi_{\mathrm{m}}$. The reduced capacity for fusion in this subpopulation of mitochondria was attributed to low levels of the OPA1 protein [104]. Increased autophagy levels have also been reported in Fis1-overexpressing cells, indicating the potential for both fusion and fission events in regulating autophagy signaling pathways and mitochondrial turnover [121].

Reduced autophagy has been reported in several tissues with age [122-124], and this is associated with the presence of enlarged mitochondria. In a recent study conducted by Masiero and Sandri [125], muscle-specific knockout of the autophagy-related protein 7 (Atg7) resulted in muscle loss and weakness associated with abnormal mitochondria and the accumulation of protein aggregates and vacuoles. Impaired glucose homeostasis and abnormal mitochondrial structure were also observed in Atg7-deficient pancreatic $\beta$ cells [126]. Furthermore, electron micrographs of muscle fibers from obese and type 2 diabetic subjects revealed that mitochondria are smaller and contain abnormal inner membrane structure when compared to muscle form lean subjects $[83,98]$. Additionally, the presence of vacuole-like structures which, when stained with a membrane marker, contain fragmented mitochondria could be detected [99]. Whether these vacuoles represent autophagosomes engulfing damaged or energy-deficient mitochondria still requires con-firmation. These studies suggest that the clearance of damaged proteins and organelles such as mitochondria is vital for maintaining cellular integrity. Whether the dysregulation of autophagy contributes to the pathogenesis of type 2 diabetes remains to be investigated.

\section{Mitochondrial-Mediated Therapeutic Interventions}

As mentioned above, factors involved in mitochondrial biogenesis are vital for tissue-specific metabolic control, and recent research has focused on modalities that can improve mitochondrial function and substrate oxidation by stimulating mitochondrial biogenesis and nutrient-sensing pathways. These include physiological and macronutrient interventions, as well as pharmacological interventions.

\subsection{Physiological and Macronutrient Interventions}

4.1.1. Exercise and Weight Loss. Skeletal muscle from obese and type 2 diabetic individuals is characterized by an impaired oxidative capacity and increased IMCL content $[127,128]$. Recent studies to identify potential therapeutic modalities in obese/type 2 diabetic individuals have shown the effectiveness of both acute and chronic exercise to increase muscle glucose disposal, fatty acid oxidation, and mitochondrial biogenesis [129-131]. These changes are mediated by key metabolic factors such as AMPK and SIRT1 that are stimulated with reduced energy states and directly activate the PGC- $1 \alpha$-mediated induction of target genes, including NRF-1, Tfam, and Mfn2, as well as genes involved in glucose and fatty acid oxidation $[69,95,96,130]$. Obese and type 2 diabetic individuals have impaired activation of these signaling pathways, and their responses are attenuated when compared to healthy subjects suggesting that a higher intensity and/or duration of exercise is required to achieve the same adaptations in these patients $[130,132]$.

Weight loss alone is associated with a reduction in IMCL content $[20,133]$. However, the beneficial effects of exercise on insulin resistance occur in the absence of a change in lipid content and active lipid intermediates (e.g., ceramides, DAGs, CoAs) and in some cases even elevated IMCL levels $[134,135]$. In a study conducted by He et al. [136] using a combination of weight loss and moderateintensity exercise, increased insulin sensitivity was associated with a reduction in the size of the lipid droplets within skeletal muscle and not the total amount of lipid. The reduced size of the lipid droplets was linked to an improved aerobic capacity, suggesting that increased oxidative enzyme activity resulting from physical activity allowed for the more efficient oxidation of lipids. This is consistent with studies of trained athletes that have been reported to have higher IMCL levels, coinciding with greater oxidative enzyme capacity and improved insulin sensitivity [137, 138]. However, the adaptations that occur in patients with obesity and type 2 diabetes following these interventions do not result in an increased mtDNA content that is typical of mitochondrial biogenesis in healthy muscle. In addition, mitochondria from these individuals display abnormal morphology with altered inner membrane cristae structure [45]. These results are in keeping with the observation that the induction of mitochondrial biogenesis in patients with this metabolic 
condition differs from the molecular processes typically observed in healthy individuals.

4.1.2. Macronutrient Modulation. The beneficial effects of nutritional interventions on health and lifespan have been known for decades. Nutrient deprivation by way of CR (20$40 \%$ reduction) increases lifespan in a number of species ranging from insects to mammals $[139,140]$. The efficacy of CR in muscle is mediated by a variety of cellular and molecular changes including increased mitochondrial biogenesis and reduced metabolic rate and ROS levels, as well as increased mitochondrial autophagy [141-144]. Additionally, CR alters substrate utilization in muscle from carbohydrate metabolism towards a greater fatty acid oxidation [145]. The effects of CR have also been reported in humans albeit to a lesser extent than in animals. Civitarese et al. showed that 6 months of $25 \%$ CR in healthy young individuals resulted in increased mitochondrial biogenesis that was associated with higher levels of SIRT1, PGC- $1 \alpha$, and Tfam protein. Skeletal muscle from these subjects displayed increased mtDNA content along with decreased levels of DNA damage [146]. CR $(25 \%)$ has also been shown to improve insulin sensitivity and increase mitochondrial density and oxidative enzyme activity in skeletal muscle from type 2 diabetic individuals [131]. Furthermore, prolonged CR in obese individuals with type 2 diabetes decreased myocardial triglyceride content that was associated with improved myocardial function [147]. Thus, CR may be an effective way to increase mitochondrial biogenesis in a wide range of species and tissues; however, more studies are required to characterize its role in insulin signaling in muscle from type 2 diabetics.

\subsubsection{Pharmacological Stimulation of Mitochondrial Biogen-} esis. In an effort to reduce the severity of insulin resistance and the clinical phenotype associated with type 2 diabetes, different classes of drugs have been developed that stimulate/induce mitochondrial biogenesis and morphogenesis. Peroxisome proliferator-activated receptors (PPARs) are a family of nuclear hormone receptors that mediate the expression of a wide array of genes involved in glucose and fat metabolism. Modulation of the PPAR-activated pathway may have therapeutic potential for metabolic disorders. For example, thiazolidinediones (TZDs), such as rosiglitazone, troglitazone, and pioglitazone, are classified as PPAR $y$ agonists that improve insulin sensitivity by increasing substrate metabolism and reducing FFA levels and ROS production $[148,149]$. The PPAR agonist bezafibrate has been shown to increase mitochondrial enzyme activity, as well as the levels of several respiratory chain subunit proteins in human fibroblasts with respiratory chain deficiency [150].

Another class of drugs gaining wide acceptance in the treatment of diabetes are sirtuin 1 (SIRT1) activators. Transgenic mice overexpressing SIRT1 display lower wholebody energy requirements and have decreased rates of oxygen consumption resulting in higher metabolic efficiency when compared to their wild-type littermates. Additionally, these animals display decreased susceptibility to the development of diabetes [63]. The therapeutic potential of resveratrol, a polyphenolic extract from grape skins and red wine, has been gaining increasing popularity as a CR mimetic due to its ability to induce mitochondrial biogenesis via SIRT1mediated activation of PGC- $1 \alpha$ in mice. Furthermore, these mice have improved insulin sensitivity and are refractory to high-fat diet-induced obesity $[151,152]$. Small SIRT1 activator molecules appear to mimic the effect of CR by altering the expression of target genes involved in substrate metabolism and antioxidant defenses thereby improving metabolic function [151, 152]. In a recent study by Milne et al. [153], obese animals treated with small SIRT1 activator molecules were shown to display enhanced mitochondrial function and improved insulin sensitivity, indicating the potential use of these drugs in the treatment of type 2 diabetes.

Another group of mitochondrial biogenesis-stimulating agents showing therapeutic promise are AMPK activators such as 5 -aminoimidazole-4-carboxamide- $1-\beta$-D-ribofuranoside (AICAR) and metformin. The addition of AICAR to $\mathrm{C}_{2} \mathrm{C}_{12}$ myoblasts upregulated PGC- $1 \alpha$ gene expression through SIRT1 [154], at least in part via transcriptional activation [155]. The administration of AICAR or metformin improved insulin resistance and delayed the onset of diabetes $[156,157]$. Furthermore, metformin has been shown to improve insulin sensitivity and has been consistently used as an antidiabetic agent [158]. Thus, AMPK activators have shown great promise as a pharmacological intervention for type 2 diabetes, and future studies in this area will further our understanding of AMPK signaling pathways and promote the development of additional potentially effective antidiabetic agents.

\section{Concluding Remarks}

It is well established that mitochondria are intricately involved in the pathogenesis of type 2 diabetes. This is because of the instrumental role that mitochondria play in lipid and carbohydrate metabolism, their morphological and functional plasticity in response to inactivity and disease, and their involvement in apoptosis and autophagy. Whether the alterations observed in mitochondrial function are a cause, or a consequence, of insulin resistance and type 2 diabetes remains under debate. Despite the fact that there is conflicting evidence regarding the extent of mitochondrial dysfunction in type 2 diabetics, there is a consensus among studies that skeletal muscle mitochondria from diabetic animals or humans exhibit impairments in key transcriptional regulators. In addition, alterations in genes involved in mitochondrial fusion and fission, as well as aberrant mitochondrial morphology, are commonly associated with the increased production of ROS and the accumulation of damaged DNA, proteins, and lipids. Collectively, these transcriptional alterations can impair insulin signaling pathways leading to insulin resistance and the development of type 2 diabetes (Figure 3 ). The potential to stimulate mitochondrial biogenesis and morphogenesis through physiological interventions such as exercise, $\mathrm{CR}$, or pharmacological mimetics of mitochondrial biogenesis has shown great promise in improving insulin sensitivity and 


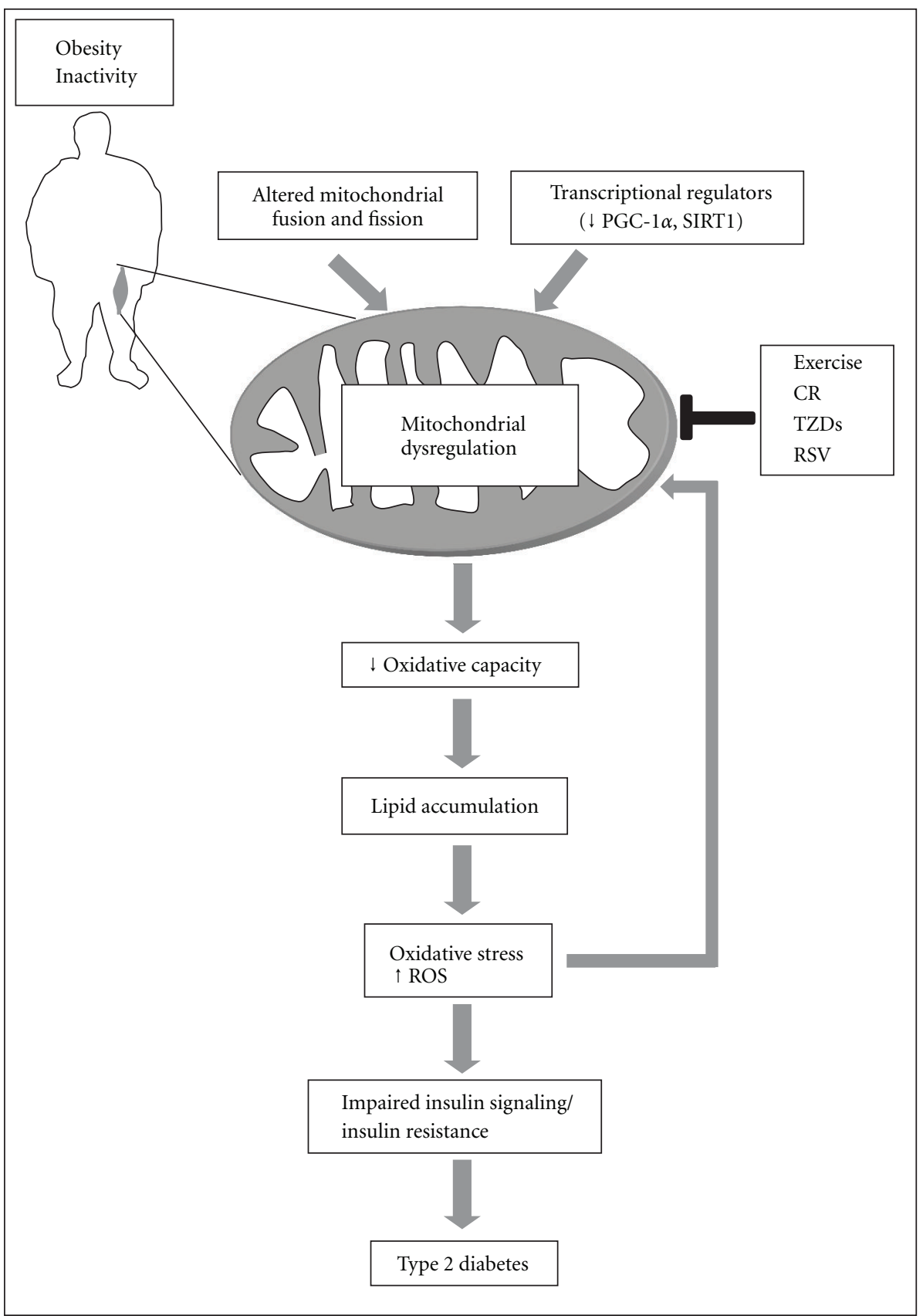

FIGURE 3: Simplified scheme that illustrates the role of mitochondrial dysregulation in the pathogenesis of type 2 diabetes in skeletal muscle. Obesity and physical inactivity can result in mitochondrial dysregulation through alterations in crucial transcriptional activators (e.g., PGC$1 \alpha$ and SIRT1), as well as impaired fusion and fission leading to aberrant mitochondrial morphology. These changes can subsequently lead to reduced oxidative capacity and cause lipid metabolite accumulation, increased oxidative stress, and the production of reactive oxygen species (ROS). Over time, the accumulation of ROS can damage DNA, proteins, and lipids, further exacerbating mitochondrial dysfunction. Collectively, these factors contribute to impaired insulin signaling pathways and increase the risk of type 2 diabetes. On the other hand, physiological interventions, including exercise and caloric restriction (CR), as well as pharmacological agents such as thiazolidinediones (TZDs) and resveratrol (RSV) have been shown to stimulate mitochondrial biogenesis and reduce mitochondrial dysfunction that is observed with type 2 diabetes in muscle.

attenuating the clinical phenotype associated with these metabolic disorders. Thus, the closer we come to understanding the molecular mechanisms governing these pathways during normal physiological conditions, the better chance we have to develop new therapeutic strategies that may one day prevent or treat type 2 diabetes.

\section{Acknowledgments}

This work was supported by the Institut de Cardiologie de Québec grant to D. R. Joanisse and R. G. Baillot and grants from the Natural Sciences and Engineering Research Council of Canada (NSERC) and the Canadian Institutes of Health 
Research to D. A. Hood. A. M. Joseph was a recipient of an Ontario Graduate Scholarship (OGS). D. A. Hood is a Canada Research Chair in Cell Physiology.

\section{References}

[1] M. E. Patti and S. Corvera, "The role of mitochondria in the pathogenesis of type 2 diabetes," Endocrine Reviews, vol. 31, no. 3, pp. 364-395, 2010.

[2] K. F. Petersen and G. I. Shulman, "Etiology of insulin resistance," The American Journal of Medicine, vol. 119, no. 5, supplement 1, pp. S10-S16, 2006.

[3] E. W. Kraegen, P. W. Clark, A. B. Jenkins, E. A. Daley, D. J. Chisholm, and L. H. Storlien, "Development of muscle insulin resistance after liver insulin resistance in high-fat-fed rats," Diabetes, vol. 40, no. 11, pp. 1397-1403, 1991.

[4] J. C. Russell, G. Shillabeer, J. Bar-Tana et al., "Development of insulin resistance in the JCR:LA-cp rat: role of triacylglycerols and effects of MEDICA 16," Diabetes, vol. 47, no. 5, pp. 770$778,1998$.

[5] A. Y. Seo, A. M. Joseph, D. Dutta, J. C. Y. Hwang, J. P. Aris, and C. Leeuwenburgh, "New insights into the role of mitochondria in aging: mitochondrial dynamics and more," Journal of Cell Science, vol. 123, part 15, pp. 2533-2542, 2010.

[6] Y. Yoon, C. A. Galloway, B. S. Jhun, and T. Yu, "Mitochondrial dynamics in diabetes," Antioxidants and Redox Signaling, vol. 14, no. 3, pp. 439-457, 2011.

[7] D. E. Kelley, B. Goodpaster, R. R. Wing, and J. A. Simoneau, "Skeletal muscle fatty acid metabolism in association with insulin resistance, obesity, and weight loss," American Journal of Physiology, vol. 277, no. 6, part 1, pp. E1130-E1141, 1999.

[8] T. R. Koves, J. R. Ussher, R. C. Noland et al., "Mitochondrial overload and incomplete fatty acid oxidation contribute to skeletal muscle insulin resistance," Cell Metabolism, vol. 7, no. 1, pp. 45-56, 2008.

[9] S. Lillioja, A. A. Young, C. L. Culter et al., "Skeletal muscle capillary density and fiber type are possible determinants of in vivo insulin resistance in man," Journal of Clinical Investigation, vol. 80, no. 2, pp. 415-424, 1987.

[10] M. Mogensen, K. Sahlin, M. Fernström et al., "Mitochondrial respiration is decreased in skeletal muscle of patients with type 2 diabetes," Diabetes, vol. 56, no. 6, pp. 1592-1599, 2007.

[11] D. J. Dyck, S. J. Peters, J. Glatz et al., "Functional differences in lipid metabolism in resting skeletal muscle of various fiber types," American Journal of Physiology, vol. 272, no. 3, part 1, pp. E340-E351, 1997.

[12] E. J. Anderson, H. Yamazaki, and P. D. Neufer, "Induction of endogenous uncoupling protein 3 suppresses mitochondrial oxidant emission during fatty acid-supported respiration," Journal of Biological Chemistry, vol. 282, no. 43, pp. 3125731266, 2007.

[13] R. A. DeFronzo, E. Jacot, E. Jequier, E. Maeder, J. Wahren, and J. P. Felber, "The effect of insulin on the disposal of intravenous glucose: results from indirect calorimetry and hepatic and femoral venous catheterization," Diabetes, vol. 30, no. 12, pp. 1000-1007, 1981.

[14] R. A. DeFronzo, R. Gunnarsson, and O. Bjorkman, "Effects of insulin on peripheral and splanchnic glucose metabolism in noninsulin-dependent (type II) diabetes mellitus," Journal of Clinical Investigation, vol. 76, no. 1, pp. 149-155, 1985.

[15] F. R. Jornayvaz and G. I. Shulman, "Regulation of mitochondrial biogenesis," Essays in Biochemistry, vol. 47, pp. 69-84, 2010.
[16] I. R. Lanza and K. Sreekumaran Nair, "Regulation of skeletal muscle mitochondrial function: genes to proteins," Acta Physiologica, vol. 199, no. 4, pp. 529-547, 2010.

[17] D. A. Hood, M. Takahashi, M. K. Connor, and D. Freyssenet, "Assembly of the cellular powerhouse: current issues in muscle mitochondrial biogenesis," Exercise and Sport Sciences Reviews, vol. 28, no. 2, pp. 68-73, 2000.

[18] V. Ljubicic, A. M. Joseph, A. Saleem et al., "Transcriptional and post-transcriptional regulation of mitochondrial biogenesis in skeletal muscle: effects of exercise and aging," Biochimica et Biophysica Acta, vol. 1800, no. 3, pp. 223-234, 2010.

[19] D. E. Kelley, K. V. Williams, and J. C. Price, "Insulin regulation of glucose transport and phosphorylation in skeletal muscle assessed by PET," American Journal of Physiology, vol. 277, no. 2, part 1, pp. E361-E369, 1999.

[20] B. H. Goodpaster, R. Theriault, S. C. Watkins, and D. E. Kelley, "Intramuscular lipid content is increased in obesity and decreased by weight loss," Metabolism: Clinical and Experimental, vol. 49, no. 4, pp. 467-472, 2000.

[21] K. F. Petersen, D. Befroy, S. Dufour et al., "Mitochondrial dysfunction in the elderly: possible role in insulin resistance," Science, vol. 300, no. 5622, pp. 1140-1142, 2003.

[22] K. Morino, K. F. Petersen, S. Dufour et al., "Reduced mitochondrial density and increased IRS- 1 serine phosphorylation in muscle of insulin-resistant offspring of type 2 diabetic parents," Journal of Clinical Investigation, vol. 115, no. 12, pp. 3587-3593, 2005.

[23] M. Gaster, A. C. Rustan, V. Aas, and H. Beck-Nielsen, "Reduced lipid oxidation in skeletal muscle from type 2 diabetic subjects may be of genetic origin: evidence from cultured myotubes," Diabetes, vol. 53, no. 3, pp. 542-548, 2004.

[24] J. M. W. van den Ouweland, H. H. P. J. Lemkes, W. Ruitenbeek et al., "Mutation in mitochondrial tRMALeu(UUR) gene in a large pedigree with maternally transmitted type II diabetes mellitus and deafness," Nature Genetics, vol. 1, no. 5, pp. 368-371, 1992.

[25] V. K. Mootha, C. M. Lindgren, K. F. Eriksson et al., "PGC$1 \alpha$-responsive genes involved in oxidative phosphorylation are coordinately downregulated in human diabetes," Nature Genetics, vol. 34, no. 3, pp. 267-273, 2003.

[26] M. E. Patti, A. J. Butte, S. Crunkhorn et al., "Coordinated reduction of genes of oxidative metabolism in humans with insulin resistance and diabetes: potential role of $P G C 1$ and NRF1," Proceedings of the National Academy of Sciences of the United States of America, vol. 100, no. 14, pp. 8466-8471, 2003.

[27] R. C. Scarpulla, "Nuclear control of respiratory chain expression in mammalian cells," Journal of Bioenergetics and Biomembranes, vol. 29, no. 2, pp. 109-119, 1997.

[28] N. Lenka, C. Vijayasarathy, J. Mullick, and N. G. Avadhani, "Structural organization and transcription regulation of nuclear genes encoding the mammalian cytochrome $\mathrm{c}$ oxidase complex," Progress in Nucleic Acid Research and Molecular Biology, vol. 61, pp. 309-344, 1998.

[29] Z. Wu, P. Puigserver, U. Andersson et al., "Mechanisms controlling mitochondrial biogenesis and respiration through the thermogenic coactivator PGC-1," Cell, vol. 98, no. 1, pp. 115-124, 1999.

[30] A. Zaid, R. Li, K. Luciakova, P. Barath, S. Nery, and B. D. Nelson, "On the role of the general transcription factor $\mathrm{Sp} 1$ in the activation and repression of diverse mammalian 
oxidative phosphorylation genes," Journal of Bioenergetics and Biomembranes, vol. 31, no. 2, pp. 129-135, 1999.

[31] M. J. Evans and R. C. Scarpulla, "NRF-1: a trans-activator of nuclear-encoded respiratory genes in animal cells," Genes and Development, vol. 4, no. 6, pp. 1023-1034, 1990.

[32] J. R. Blesa, J. M. Hernández, and J. Hernández-Yago, "NRF2 transcription factor is essential in promoting human Tomm70 gene expression," Mitochondrion, vol. 3, no. 5, pp. 251-259, 2004.

[33] N. Gleyzer, K. Vercauteren, and R. C. Scarpulla, "Control of mitochondrial transcription specificity factors (TFB1M and TFB2M) by nuclear respiratory factors (NRF-1 and NRF2) and PGC-1 family coactivators," Molecular and Cellular Biology, vol. 25, no. 4, pp. 1354-1366, 2005.

[34] J. J. Lehman, P. M. Barger, A. Kovacs, J. E. Saffitz, D. M. Medeiros, and D. P. Kelly, "Peroxisome proliferator-activated receptor $\gamma$ coactivator-1 promotes cardiac mitochondrial biogenesis," Journal of Clinical Investigation, vol. 106, no. 7, pp. 847-856, 2000.

[35] T. Wenz, S. G. Rossi, R. L. Rotundo, B. M. Spiegelman, and C. T. Moraes, "Increased muscle PGC- $1 \alpha$ expression protects from sarcopenia and metabolic disease during aging," Proceedings of the National Academy of Sciences of the United States of America, vol. 106, no. 48, pp. 20405-20410, 2009.

[36] J. Lin, H. Wu, P. T. Tarr et al., "Transcriptional co-activator PGC- $1 \alpha$ drives the formation of slow-twitch muscle fibres," Nature, vol. 418, no. 6899, pp. 797-801, 2002.

[37] H. Wu, S. B. Kanatous, F. A. Thurmond et al., "Regulation of mitochondrial biogenesis in skeletal muscle by CaMK," Science, vol. 296, no. 5566, pp. 349-352, 2002.

[38] H. Pilegaard, B. Saltin, and D. P. Neufer, "Exercise induces transient transcriptional activation of the PGC- $1 \alpha$ gene in human skeletal muscle," Journal of Physiology, vol. 546, supplement 3, pp. 851-858, 2003.

[39] D. J. Mahoney, G. Parise, S. Melov, A. Safdar, and M. A. Tarnopolsky, "Analysis of global mRNA expression in human skeletal muscle during recovery from endurance exercise," The FASEB Journal, vol. 19, no. 11, pp. 1498-1500, 2005.

[40] D. C. Wright, D. H. Han, P. M. Garcia-Roves, P. C. Geiger, T. E. Jones, and J. O. Holloszy, "Exercise-induced mitochondrial biogenesis begins before the increase in muscle PGC- $1 \alpha$ expression," Journal of Biological Chemistry, vol. 282, no. 1, pp. 194-199, 2007.

[41] J. Ek, G. Andersen, S. A. Urhammer et al., "Studies of the Pro12Ala polymorphism of the peroxisome proliferatoractivated receptor- $\gamma 2$ (PPAR- $\gamma 2$ ) gene in relation to insulin sensitivity among glucose tolerant caucasians," Diabetologia, vol. 44, no. 9, pp. 1170-1176, 2001.

[42] J. H. Kim, H. D. Shin, B. L. Park et al., "Peroxisome proliferator-activated receptor gamma coactivator 1 alpha promoter polymorphisms are associated with early-onset type 2 diabetes mellitus in the Korean population," Diabetologia, vol. 48, no. 7, pp. 1323-1330, 2005.

[43] L. F. Michael, Z. Wu, R. B. Cheatham et al., "Restoration of insulin-sensitive glucose transporter (GLUT4) gene expression in muscle cells by the transcriptional coactivator PGC1," Proceedings of the National Academy of Sciences of the United States of America, vol. 98, no. 7, pp. 3820-3825, 2001.

[44] J. P. Silva, M. Köhler, C. Graff et al., "Impaired insulin secretion and $\beta$-cell loss in tissue-specific knockout mice with mitochondrial diabetes," Nature Genetics, vol. 26, no. 3, pp. 336-340, 2000.
[45] E. V. Menshikova, V. B. Ritov, R. E. Ferrell, K. Azuma, B. H. Goodpaster, and D. E. Kelley, "Characteristics of skeletal muscle mitochondrial biogenesis induced by moderateintensity exercise and weight loss in obesity," Journal of Applied Physiology, vol. 103, no. 1, pp. 21-27, 2007.

[46] G. P. Holloway, C. G. R. Perry, A. B. Thrush et al., "PGC-1 $\alpha$ 's relationship with skeletal muscle palmitate oxidation is not present with obesity despite maintained PGC- $1 \alpha$ and PGC$1 \beta$ protein," American Journal of Physiology, vol. 105, no. 22, pp. 7815-7820, 2008.

[47] C. Handschin, S. C. Cheol, S. Chin et al., "Abnormal glucose homeostasis in skeletal muscle-specific PGC- $1 \alpha$ knockout mice reveals skeletal muscle-pancreatic $\beta$ cell crosstalk," Journal of Clinical Investigation, vol. 117, no. 11, pp. 34633474, 2007.

[48] C. Zechner, L. Lai, J. F. Zechner et al., "Total skeletal muscle PGC-1 deficiency uncouples mitochondrial derangements from fiber type determination and insulin sensitivity," Cell Metabolism, vol. 12, no. 6, pp. 633-642, 2010.

[49] K. Aquilano, P. Vigilanza, S. Baldelli, B. Pagliei, G. Rotilio, and M. R. Ciriolo, "Peroxisome proliferator-activated receptor $\gamma$ co-activator $1 \alpha(\mathrm{PGC}-1 \alpha)$ and sirtuin 1 (SIRT1) reside in mitochondria: possible direct function in mitochondrial biogenesis," Journal of Biological Chemistry, vol. 285, no. 28, pp. 21590-21599, 2010.

[50] A. Safdar, J. P. Little, A. J. Stokl, B. P. Hettinga, M. Akhtar, and M. A. Tarnopolsky, "Exercise increases mitochondrial PGC$1 \alpha$ content and promotes nuclear-mitochondrial cross-talk to coordinate mitochondrial biogenesis," Journal of Biological Chemistry, vol. 286, no. 12, pp. 10605-10617, 2011.

[51] D. Chen and L. Guarente, "SIR2: a potential target for calorie restriction mimetics," Trends in Molecular Medicine, vol. 13, no. 2, pp. 64-71, 2007.

[52] J. Y. Huang, M. D. Hirschey, T. Shimazu, L. Ho, and E. Verdin, "Mitochondrial sirtuins," Biochimica et Biophysica Acta, vol. 1804, no. 8, pp. 1645-1651, 2010.

[53] S. Nemoto, M. M. Fergusson, and T. Finkel, "Nutrient availability regulates SIRT1 through a forkhead-dependent pathway," Science, vol. 306, no. 5704, pp. 2105-2108, 2004.

[54] D. Chen, J. Bruno, E. Easlon et al., "Tissue-specific regulation of SIRT1 by calorie restriction," Genes and Development, vol. 22, no. 13, pp. 1753-1757, 2008.

[55] H. S. Kwon and M. Ott, "The ups and downs of SIRT1," Trends in Biochemical Sciences, vol. 33, no. 11, pp. 517-525, 2008.

[56] M. Suwa, H. Nakano, Z. Radak, and S. Kumagai, "Endurance exercise increases the SIRT1 and peroxisome proliferatoractivated receptor $\gamma$ coactivator- $1 \alpha$ protein expressions in rat skeletal muscle," Metabolism: Clinical and Experimental, vol. 57, no. 7, pp. 986-998, 2008.

[57] T. Finkel, C. X. Deng, and R. Mostoslavsky, "Recent progress in the biology and physiology of sirtuins," Nature, vol. 460, no. 7255, pp. 587-591, 2009.

[58] L. Bordone, D. Cohen, A. Robinson et al., "SIRT1 transgenic mice show phenotypes resembling calorie restriction," Aging Cell, vol. 6, no. 6, pp. 759-767, 2007.

[59] D. Chen, A. D. Steele, S. Lindquist, and L. Guarente, "Medicine: increase in activity during calorie restriction requires Sirt1," Science, vol. 310, no. 5754, p. 1641, 2005.

[60] N. Makino, T. Maeda, J. I. Oyama, Y. Higuchi, and K. Mimori, "Improving insulin sensitivity via activation of PPAR- $\gamma$ increases telomerase activity in the heart of OLETF rats," American Journal of Physiology, vol. 297, no. 6, pp. H2188H2195, 2009. 
[61] A. R. Subauste and C. F. Burant, "Role of FoxO1 in FFAinduced oxidative stress in adipocytes," American Journal of Physiology, vol. 293, no. 1, pp. E159-E164, 2007.

[62] S. B. Pedersen, J. Ølholm, S. K. Paulsen, M. F. Bennetzen, and B. Richelsen, "Low Sirt1 expression, which is upregulated by fasting, in human adipose tissue from obese women," International Journal of Obesity, vol. 32, no. 8, pp. 1250-1255, 2008.

[63] A. S. Banks, N. Kon, C. Knight et al., "SirT1 gain of function increases energy efficiency and prevents diabetes in mice," Cell Metabolism, vol. 8, no. 4, pp. 333-341, 2008.

[64] N. Fujii, T. Hayashi, M. F. Hirshman et al., "Exercise induces isoform-specific increase in 5'AMP-activated protein kinase activity in human skeletal muscle," Biochemical and Biophysical Research Communications, vol. 273, no. 3, pp. 1150-1155, 2000.

[65] O. M. Palacios, J. J. Carmona, S. Michan et al., "Diet and exercise signals regulate SIRT3 and activate AMPK and PGC1alpha in skeletal muscle," Aging, vol. 1, no. 9, pp. 771-783, 2009.

[66] S. Nemoto, M. M. Fergusson, and T. Finkel, "SIRT1 functionally interacts with the metabolic regulator and transcriptional coactivator PGC-1 $\alpha$," Journal of Biological Chemistry, vol. 280, no. 16, pp. 16456-16460, 2005.

[67] J. T. Rodgers, C. Lerin, W. Haas, S. P. Gygi, B. M. Spiegelman, and P. Puigserver, "Nutrient control of glucose homeostasis through a complex of PGC- $1 \alpha$ and SIRT1," Nature, vol. 434, no. 7029, pp. 113-118, 2005.

[68] Z. Gerhart-Hines, J. T. Rodgers, O. Bare et al., "Metabolic control of muscle mitochondrial function and fatty acid oxidation through SIRT1/PGC-1 $\alpha$," The EMBO Journal, vol. 26, no. 7, pp. 1913-1923, 2007.

[69] S. Jager, C. Handschin, J. St-Pierre, and B. M. Spiegelman, "AMP-activated protein kinase (AMPK) action in skeletal muscle via direct phosphorylation of PGC-1 $\alpha$," Proceedings of the National Academy of Sciences of the United States of America, vol. 104, no. 29, pp. 12017-12022, 2007.

[70] W. Qiang, K. Weiqiang, Z. Qing, Z. Pengju, and L. Yi, "Aging impairs insulin-stimulated glucose uptake in rat skeletal muscle via suppressing AMPK $\alpha$," Experimental and Molecular Medicine, vol. 39, no. 4, pp. 535-543, 2007.

[71] R. M. Reznick, H. Zong, J. Li et al., "Aging-Associated Reductions in AMP-Activated Protein Kinase Activity and Mitochondrial Biogenesis," Cell Metabolism, vol. 5, no. 2, pp. 151-156, 2007.

[72] I. R. Lanza, D. K. Short, K. R. Short et al., "Endurance exercise as a countermeasure for aging," Diabetes, vol. 57, no. 11, pp. 2933-2942, 2008.

[73] S. Someya, W. Yu, W. C. Hallows et al., "Sirt3 mediates reduction of oxidative damage and prevention of age-related hearing loss under caloric restriction," Cell, vol. 143, no. 5, pp. 802-812, 2010.

[74] X. Kong, R. Wang, Y. Xue et al., "Sirtuin 3, a new target of PGC- $1 \alpha$, plays an important role in the suppression of ROS and mitochondrial biogenesis," PLoS ONE, vol. 5, no. 7, Article ID e11707, 2010.

[75] D. A. Hood, "Invited review: contractile activity-induced mitochondrial biogenesis in skeletal muscle," Journal of Applied Physiology, vol. 90, no. 3, pp. 1137-1157, 2001.

[76] A. M. Cogswell, R. J. Stevens, and D. A. Hood, "Properties of skeletal muscle mitochondria isolated from subsarcolemmal and intermyofibrillar regions," American Journal of Physiology, vol. 264, no. 2, part 1, pp. C383-C389, 1993.
[77] V. B. Ritov, E. V. Menshikova, J. He, R. E. Ferrell, B. H. Goodpaster, and D. E. Kelley, "Deficiency of subsarcolemmal mitochondria in obesity and type 2 diabetes," Diabetes, vol. 54, no. 1, pp. 8-14, 2005.

[78] M. Takahashi and D. A. Hood, "Protein import into subsarcolemmal and intermyofibrillar skeletal muscle mitochondria: differential import regulation in distinct subcellular regions," Journal of Biological Chemistry, vol. 271, no. 44, pp. 27285-27291, 1996.

[79] P. J. Adhihetty, V. Ljubicic, K. J. Menzies, and D. A. Hood, "Differential susceptibility of subsarcolemmal and intermyofibrillar mitochondria to apoptotic stimuli," American Journal of Physiology, vol. 289, no. 4, pp. C994-C1001, 2005.

[80] H. Chen and D. C. Chan, "Physiological functions of mitochondrial fusion," Annals of the New York Academy of Sciences, vol. 1201, pp. 21-25, 2010.

[81] H. Bo, Y. Zhang, and L. L. Ji, "Redefining the role of mitochondria in exercise: a dynamic remodeling," Annals of the New York Academy of Sciences, vol. 1201, pp. 121-128, 2010.

[82] H. Otera and K. Mihara, "Molecular mechanisms and physiologic functions of mitochondrial dynamics," Journal of Biochemistry, vol. 149, no. 3, pp. 241-251, 2011.

[83] D. Bach, S. Pich, F. X. Soriano et al., "Mitofusin-2 determines mitochondrial network architecture and mitochondrial metabolism: a novel regulatory mechanism altered in obesity," Journal of Biological Chemistry, vol. 278, no. 19, pp. 17190-17197, 2003.

[84] M. Liesa, M. Palacín, and A. Zorzano, "Mitochondrial dynamics in mammalian health and disease," Physiological Reviews, vol. 89, no. 3, pp. 799-845, 2009.

[85] S. Züchner, I. V. Mersiyanova, M. Muglia et al., "Mutations in the mitochondrial GTPase mitofusin 2 cause Charcot-MarieTooth neuropathy type 2A," Nature Genetics, vol. 36, no. 5, pp. 449-451, 2004.

[86] H. M. E. Bienfait, F. Baas, J. H. T. M. Koelman et al., "Phenotype of Charcot-Marie-Tooth disease type 2," Neurology, vol. 68, no. 20, pp. 1658-1667, 2007.

[87] C. Alexander, M. Votruba, U. E. A. Pesch et al., "OPA1, encoding a dynamin-related GTPase, is mutated in autosomal dominant optic atrophy linked to chromosome 3q28," Nature Genetics, vol. 26, no. 2, pp. 211-215, 2000.

[88] C. Delettre, J. M. Griffoin, J. Kaplan et al., "Mutation spectrum and splicing variants in the OPAl gene," Human Genetics, vol. 109, no. 6, pp. 584-591, 2001.

[89] D. Arnoult, A. Grodet, Y. J. Lee, J. Estaquier, and C. Blackstone, "Release of OPA1 during apoptosis participates in the rapid and complete release of cytochrome $\mathrm{c}$ and subsequent mitochondrial fragmentation," Journal of Biological Chemistry, vol. 280, no. 42, pp. 35742-35750, 2005.

[90] S. Duvezin-Caubet, R. Jagasia, J. Wagener et al., "Proteolytic processing of OPA1 links mitochondrial dysfunction to alterations in mitochondrial morphology," Journal of Biological Chemistry, vol. 281, no. 49, pp. 37972-37979, 2006.

[91] H. Chen, M. Vermulst, Y. E. Wang et al., "Mitochondrial fusion is required for mtdna stability in skeletal muscle and tolerance of mtDNA mutations," Cell, vol. 141, no. 2, pp. 280-289, 2010.

[92] H. Chen, A. Chomyn, and D. C. Chan, "Disruption of fusion results in mitochondrial heterogeneity and dysfunction," Journal of Biological Chemistry, vol. 280, no. 28, pp. 2618526192, 2005.

[93] P. A. Parone, S. Da Druz, D. Tondera et al., "Preventing mitochondrial fission impairs mitochondrial function and 
leads to loss of mitochondrial DNA," PLoS ONE, vol. 3, no. 9, Article ID e3257, 2008.

[94] A. Malena, E. Loro, M. Di Re, I. J. Holt, and L. Vergani, "Inhibition of mitochondrial fission favours mutant over wild-type mitochondrial DNA," Human Molecular Genetics, vol. 18, no. 18, pp. 3407-3416, 2009.

[95] R. Cartoni, B. Léger, M. B. Hock et al., "Mitofusins 1/2 and ERR $\alpha$ expression are increased in human skeletal muscle after physical exercise," Journal of Physiology, vol. 567, part 1, pp. 349-358, 2005.

[96] H. Ding, N. Jiang, H. Liu et al., "Response of mitochondrial fusion and fission protein gene expression to exercise in rat skeletal muscle," Biochimica et Biophysica Acta, vol. 1800, no. 3, pp. 250-256, 2010.

[97] A. Garnier, D. Fortin, J. Zoll et al., "Coordinated changes in mitochondrial function and biogenesis in healthy and diseased human skeletal muscle," The FASEB Journal, vol. 19, no. 1, pp. 43-52, 2005.

[98] D. Bach, D. Naon, S. Pich et al., "Expression of Mfn2, the Charcot-Marie-Tooth neuropathy type 2A gene, in human skeletal muscle: effects of type 2 diabetes, obesity, weight loss, and the regulatory role of tumor necrosis factor $\alpha$ and interleukin-6," Diabetes, vol. 54, no. 9, pp. 2685-2693, 2005.

[99] D. E. Kelley, J. He, E. V. Menshikova, and V. B. Ritov, "Dysfunction of mitochondria in human skeletal muscle in type 2 diabetes," Diabetes, vol. 51, no. 10, pp. 2944-2950, 2002.

[100] S. Pich, D. Bach, P. Briones et al., "The Charcot-Marie-Tooth type 2A gene product, Mfn2, up-regulates fuel oxidation through expression of OXPHOS system," Human Molecular Genetics, vol. 14, no. 11, pp. 1405-1415, 2005.

[101] G. Mingrone, M. Manco, M. Calvani, M. Castagneto, D. Naon, and A. Zorzano, "Could the low level of expression of the gene encoding skeletal muscle mitofusin-2 account for the metabolic inflexibility of obesity?" Diabetologia, vol. 48, no. 10, pp. 2108-2114, 2005.

[102] E. Nisoli, C. Tonello, A. Cardile et al., "Cell biology: calorie restriction promotes mitochondrial biogenesis by inducing the expression of eNOS," Science, vol. 310, no. 5746, pp. 314317, 2005.

[103] F. X. Soriano, M. Liesa, D. Bach, D. C. Chan, M. Palacín, and A. Zorzano, "Evidence for a mitochondrial regulatory pathway defined by peroxisome proliferator-activated receptor$\gamma$ coactivator- $1 \alpha$, estrogen-related receptor- $\alpha$, and mitofusin 2," Diabetes, vol. 55, no. 6, pp. 1783-1791, 2006.

[104] G. Twig, A. Elorza, A. J. A. Molina et al., "Fission and selective fusion govern mitochondrial segregation and elimination by autophagy," The EMBO Journal, vol. 27, no. 2, pp. 433-446, 2008.

[105] C. Zanna, A. Ghelli, A. M. Porcelli et al., "OPA1 mutations associated with dominant optic atrophy impair oxidative phosphorylation and mitochondrial fusion," Brain, vol. 131, part 2, pp. 352-367, 2008.

[106] P. J. Adhihetty, M. F. N. O'Leary, and D. A. Hood, "Mitochondria in skeletal muscle: adaptable rheostats of apoptotic susceptibility," Exercise and Sport Sciences Reviews, vol. 36, no. 3, pp. 116-121, 2008.

[107] T. Yu, J. L. Robotham, and Y. Yoon, "Increased production of reactive oxygen species in hyperglycemic conditions requires dynamic change of mitochondrial morphology," Proceedings of the National Academy of Sciences of the United States of America, vol. 103, no. 8, pp. 2653-2658, 2006.
[108] P. Delivani, C. Adrain, R. C. Taylor, P. J. Duriez, and S. J. Martin, "Role for CED-9 and Egl-1 as regulators of mitochondrial fission and fusion dynamics," Molecular Cell, vol. 21, no. 6, pp. 761-773, 2006.

[109] C. Brooks, Q. Wei, L. Feng et al., "Bak regulates mitochondrial morphology and pathology during apoptosis by interacting with mitofusins," Proceedings of the National Academy of Sciences of the United States of America, vol. 104, no. 28, pp. 11649-11654, 2007.

[110] D. I. James, P. A. Parone, Y. Mattenberger, and J. C. Martinou, "hFis1, a novel component of the mammalian mitochondrial fission machinery," Journal of Biological Chemistry, vol. 278, no. 38, pp. 36373-36379, 2003.

[111] S. B. Berman, Y. B. Chen, B. Qi et al., "Bcl-xL increases mitochondrial fission, fusion, and biomass in neurons," Journal of Cell Biology, vol. 184, no. 5, pp. 707-719, 2009.

[112] C. Bonnard, A. Durand, S. Peyrol et al., "Mitochondrial dysfunction results from oxidative stress in the skeletal muscle of diet-induced insulin-resistant mice," Journal of Clinical Investigation, vol. 118, no. 2, pp. 789-800, 2008.

[113] T. Nishikawa, D. Edelstein, X. L. Du et al., "Normalizing mitochondrial superoxide production blocks three pathways of hyperglycaemic damage," Nature, vol. 404, no. 6779, pp. 787-790, 2000.

[114] G. Liot, B. Bossy, S. Lubitz, Y. Kushnareva, N. Sejbuk, and E. Bossy-Wetzel, "Complex II inhibition by 3-NP causes mitochondrial fragmentation and neuronal cell death via an NMDA- and ROS-dependent pathway," Cell Death and Differentiation, vol. 16, no. 6, pp. 899-909, 2009.

[115] M. Jendrach, S. Mai, S. Pohl, M. Vöth, and J. BereiterHahn, "Short- and long-term alterations of mitochondrial morphology, dynamics and mtDNA after transient oxidative stress," Mitochondrion, vol. 8, no. 4, pp. 293-304, 2008.

[116] R. N. Baumgartner, "Body composition in healthy aging," Annals of the New York Academy of Sciences, vol. 904, pp. 437448, 2000.

[117] M. Zamboni, G. Mazzali, F. Fantin, A. Rossi, and V. Di Francesco, "Sarcopenic obesity: a new category of obesity in the elderly," Nutrition, Metabolism and Cardiovascular Diseases, vol. 18, no. 5, pp. 388-395, 2008.

[118] B. Sishi, B. Loos, B. Ellis, W. Smith, E. F. Du Toit, and A. M. Engelbrecht, "Diet-induced obesity alters signalling pathways and induces atrophy and apoptosis in skeletal muscle in a prediabetic rat model," Experimental Physiology, vol. 96, no. 2, pp. 179-193, 2011.

[119] R. T. Hepple, "Why eating less keeps mitochondria working in aged skeletal muscle," Exercise and Sport Sciences Reviews, vol. 37, no. 1, pp. 23-28, 2009.

[120] M. Sandri, "Autophagy in skeletal muscle," FEBS Letters, vol. 584, no. 7, pp. 1411-1416, 2010.

[121] L. C. Gomes and L. Scorrano, "High levels of Fis1, a profission mitochondrial protein, trigger autophagy," Biochimica et Biophysica Acta, vol. 1777, no. 7-8, pp. 860-866, 2008.

[122] A. Terman, "The effect of age on formation and elimination of autophagic vacuoles in mouse hepatocytes," Gerontology, vol. 41, supplement 2, pp. 319-326, 1995.

[123] A. Donati, G. Cavallini, C. Paradiso et al., "Age-related changes in the autophagic proteolysis of rat isolated liver cells: effects of antiaging dietary restrictions," Journals of Gerontology A, vol. 56, no. 9, pp. B375-B383, 2001.

[124] S. E. Wohlgemuth, A. Y. Seo, E. Marzetti, H. A. Lees, and C. Leeuwenburgh, "Skeletal muscle autophagy and apoptosis 
during aging: effects of calorie restriction and life-long exercise," Experimental Gerontology, vol. 45, no. 2, pp. 138$148,2010$.

[125] E. Masiero and M. Sandri, "Autophagy inhibition induces atrophy and myopathy in adult skeletal muscles," Autophagy, vol. 6, no. 2, pp. 307-309, 2010.

[126] H. S. Jung and M. S. Lee, "Role of autophagy in diabetes and mitochondria," Annals of the New York Academy of Sciences, vol. 1201, pp. 79-83, 2010.

[127] D. E. Kelley and J. A. Simoneau, "Impaired free fatty acid utilization by skeletal muscle in non-insulin- dependent diabetes mellitus," Journal of Clinical Investigation, vol. 94, no. 6, pp. 2349-2356, 1994.

[128] J. A. Simoneau and D. E. Kelley, "Altered glycolytic and oxidative capacities of skeletal muscle contribute to insulin resistance in NIDDM," Journal of Applied Physiology, vol. 83, no. 1, pp. 166-171, 1997.

[129] E. J. Henriksen, "Invited review: effects of acute exercise and exercise training on insulin resistance," Journal of Applied Physiology, vol. 93, no. 2, pp. 788-796, 2002.

[130] A. Sriwijitkamol, D. K. Coletta, E. Wajcberg et al., "Effect of acute exercise on AMPK signaling in skeletal muscle of subjects with type 2 diabetes: a time-course and doseresponse study," Diabetes, vol. 56, no. 3, pp. 836-848, 2007.

[131] F. G. S. Toledo, E. V. Menshikova, V. B. Ritov et al., "Effects of physical activity and weight loss on skeletal muscle mitochondria and relationship with glucose control in type 2 diabetes," Diabetes, vol. 56, no. 8, pp. 2142-2147, 2007.

[132] M. I. Herńandez-Alvarez, H. Thabit, N. Burns et al., "Subjects with early-onset type 2 diabetes show defective activation of the skeletal muscle PGC- $1 \alpha /$ mitofusin-2 regulatory pathway in response to physical activity," Diabetes Care, vol. 33, no. 3, pp. 645-651, 2010.

[133] A. V. Greco, G. Mingrone, A. Giancaterini et al., "Insulin resistance in morbid obesity: reversal with intramyocellular fat depletion," Diabetes, vol. 51, no. 1, pp. 144-151, 2002.

[134] P. Malenfant, A. Tremblay, É. Doucet, P. Imbeault, J. A. Simoneau, and D. R. Joanisse, "Elevated intramyocellular lipid concentration in obese subjects is not reduced after diet and exercise training," American Journal of Physiology, vol. 280, no. 4, pp. E632-E639, 2001.

[135] V. B. Schrauwen-Hinderling, P. Schrauwen, M. K. C. Hesselink et al., "The increase in intramyocellular lipid content is a very early response to training," Journal of Clinical Endocrinology and Metabolism, vol. 88, no. 4, pp. 1610-1616, 2003.

[136] J. He, B. H. Goodpaster, and D. E. Kelley, "Effects of weight loss and physical activity on muscle lipid content and droplet size," Obesity Research, vol. 12, no. 5, pp. 761-769, 2004.

[137] H. Hoppeler, H. Howald, K. Conley et al., "Endurance training in humans: aerobic capacity and structure of skeletal muscle," Journal of Applied Physiology, vol. 59, no. 2, pp. 320327, 1985.

[138] B. H. Goodpaster, J. He, S. Watkins, and D. E. Kelley, "Skeletal muscle lipid content and insulin resistance: evidence for a paradox in endurance-trained athletes," Journal of Clinical Endocrinology and Metabolism, vol. 86, no. 12, pp. 57555761, 2001.

[139] C. M. McCay, M. F. Crowell, and L. A. Maynard, "The effect of retarded growth upon the length of life span and upon the ultimate body size. 1935," Nutrition, vol. 5, no. 3, pp. 155171, 1989.

[140] R. J. Colman, R. M. Anderson, S. C. Johnson et al., "Caloric restriction delays disease onset and mortality in rhesus monkeys," Science, vol. 325, no. 5937, pp. 201-204, 2009.
[141] R. Gredilla, A. Sanz, M. Lopez-Torres, and G. Barja, "Caloric restriction decreases mitochondrial free radical generation at complex I and lowers oxidative damage to mitochondrial DNA in the rat heart," The FASEB Journal, vol. 15, no. 9, pp. 1589-1591, 2001.

[142] S. J. Lin, M. Kaeberlein, A. A. Andalis et al., "Calorie restriction extends Saccharomyces cerevisiae lifespan by increasing respiration," Nature, vol. 418, no. 6895, pp. 344-348, 2002.

[143] E. Morselli, M. C. Maiuri, M. Markaki et al., "The life spanprolonging effect of sirtuin-1 is mediated by autophagy," Autophagy, vol. 6, no. 1, pp. 186-188, 2010.

[144] N. A. Bishop and L. Guarente, "Two neurons mediate dietrestriction-induced longevity in C. elegans," Nature, vol. 447, no. 7144, pp. 545-549, 2007.

[145] M. D. Bruss, C. F. Khambatta, M. A. Ruby, I. Aggarwal, and M. K. Hellerstein, "Calorie restriction increases fatty acid synthesis and whole body fat oxidation rates," American Journal of Physiology, vol. 298, no. 1, pp. E108-E116, 2010.

[146] A. E. Civitarese, S. Carling, L. K. Heilbronn et al., "Calorie restriction increases muscle mitochondrial biogenesis in healthy humans," PLoS Medicine, vol. 4, no. 3, pp. 485-494, 2007.

[147] S. Hammer, M. Snel, H. J. Lamb et al., "Prolonged caloric restriction in obese patients with type 2 diabetes mellitus decreases myocardial triglyceride content and improves myocardial function," Journal of the American College of Cardiology, vol. 52, no. 12, pp. 1006-1012, 2008.

[148] K. F. Petersen, M. Krssak, S. Inzucchi, G. W. Cline, S. Dufour, and G. I. Shulman, "Mechanism of troglitazone action in type 2 diabetes," Diabetes, vol. 49, no. 5, pp. 827-831, 2000.

[149] M. Mensink, M. K. C. Hesselink, A. P. Russell, G. Schaart, J. P. Sels, and P. Schrauwen, "Improved skeletal muscle oxidative enzyme activity and restoration of PGC- $1 \alpha$ and $\mathrm{PPAR} \beta / \delta$ gene expression upon rosiglitazone treatment in obese patients with type 2 diabetes mellitus," International Journal of Obesity, vol. 31, no. 8, pp. 1302-1310, 2007.

[150] J. Bastin, F. Aubey, A. Rötig, A. Munnich, and F. Djouadi, "Activation of peroxisome proliferator-activated receptor pathway stimulates the mitochondrial respiratory chain and can correct deficiencies in patients' cells lacking its components," Journal of Clinical Endocrinology and Metabolism, vol. 93, no. 4, pp. 1433-1441, 2008.

[151] J. A. Baur, K. J. Pearson, N. L. Price et al., "Resveratrol improves health and survival of mice on a high-calorie diet," Nature, vol. 444, no. 7117, pp. 337-342, 2006.

[152] M. Lagouge, C. Argmann, Z. Gerhart-Hines et al., "Resveratrol improves mitochondrial function and protects against metabolic disease by activating SIRT1 and PGC- $1 \alpha$," Cell, vol. 127, no. 6, pp. 1109-1122, 2006.

[153] J. C. Milne, P. D. Lambert, S. Schenk et al., "Small molecule activators of SIRT1 as therapeutics for the treatment of type 2 diabetes," Nature, vol. 450, no. 7170, pp. 712-716, 2007.

[154] C. Cantó and J. Auwerx, "PGC-1 $\alpha$, SIRT1 and AMPK, an energy sensing network that controls energy expenditure," Current Opinion in Lipidology, vol. 20, no. 2, pp. 98-105, 2009.

[155] I. Irrcher, V. Ljubicic, A. F. Kirwan, and D. A. Hood, "AMPactivated protein kinase-regulated activation of the PGC1alpha promoter in skeletal muscle cells," PLoS ONE, vol. 3, no. 10, Article ID e3614, 2008.

[156] X. Yu, S. McCorkle, M. Wang et al., "Leptinomimetic effects of the AMP kinase activator AICAR in leptin-resistant rats: prevention of diabetes and ectopic lipid deposition," Diabetologia, vol. 47, no. 11, pp. 2012-2021, 2004. 
[157] R. Pold, L. S. Jensen, N. Jessen et al., "Long-term AICAR administration and exercise prevents diabetes in ZDF rats," Diabetes, vol. 54, no. 4, pp. 928-934, 2005.

[158] R. A. DeFronzo, "Bromocriptine: a sympatholytic, D2dopamine agonist for the treatment of type 2 diabetes," Diabetes Care, vol. 34, no. 4, pp. 789-794, 2011. 


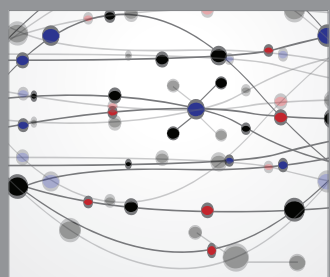

The Scientific World Journal
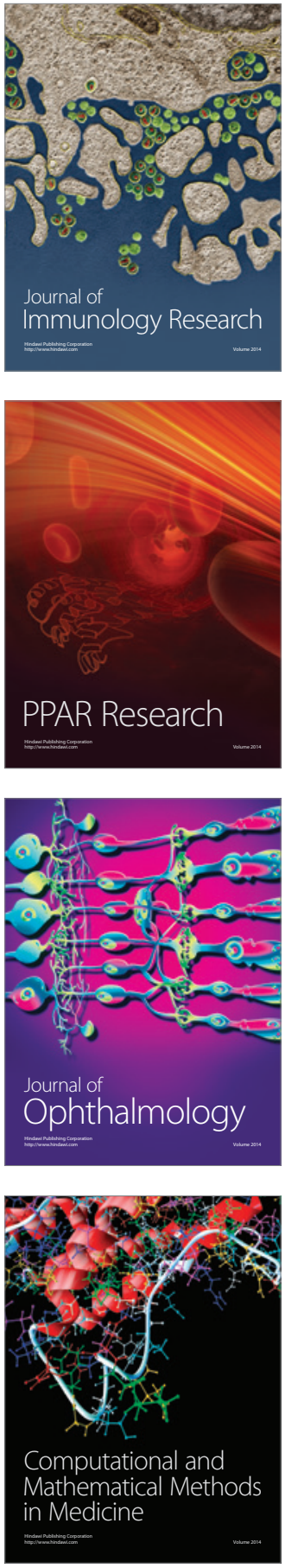

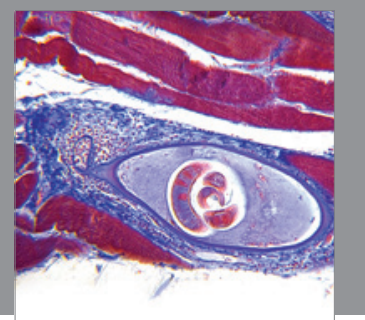

Gastroenterology

Research and Practice
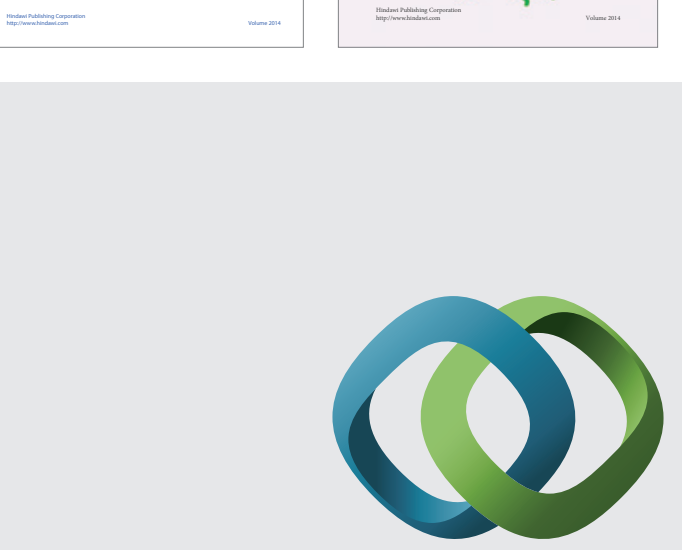

\section{Hindawi}

Submit your manuscripts at

http://www.hindawi.com
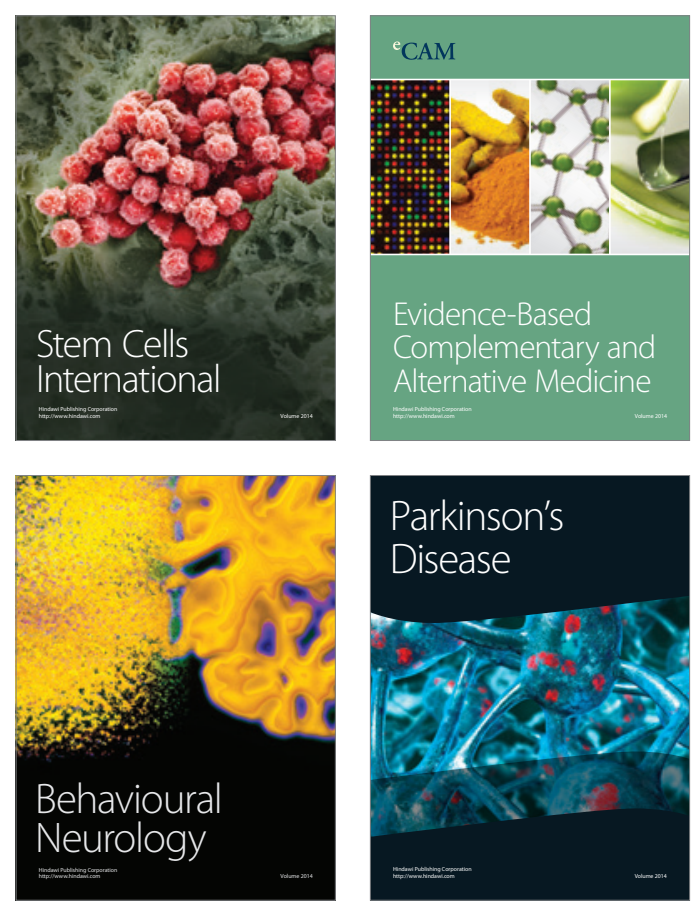

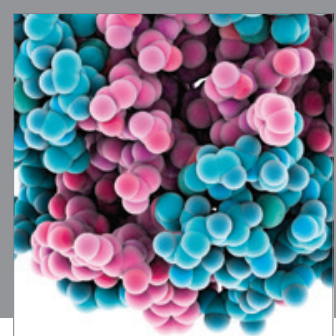

Journal of
Diabetes Research

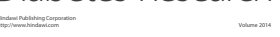

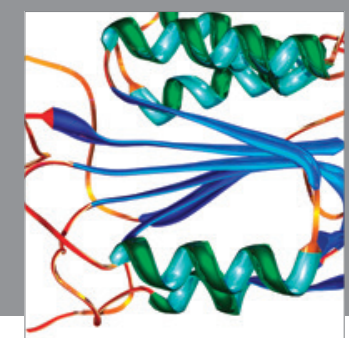

Disease Markers
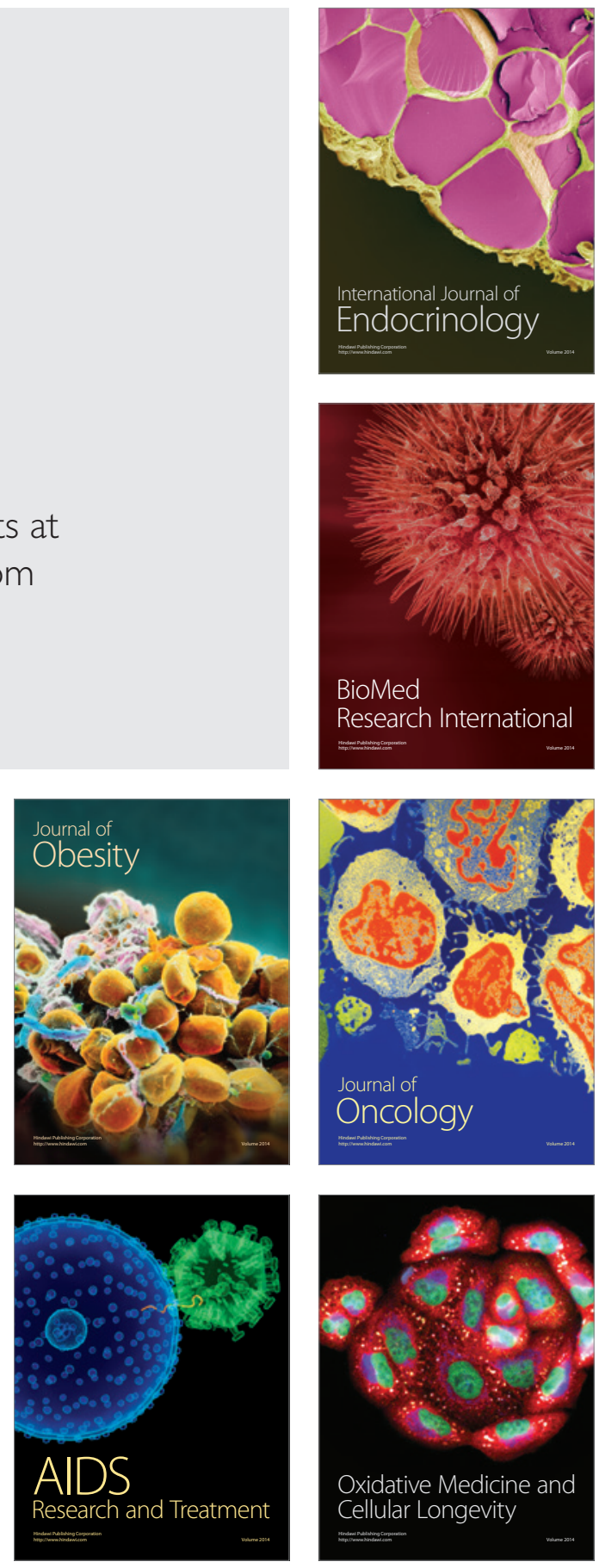